\title{
New Exact Solutions for a Higher-Order Wave Equation of KdV Type Using the Multiple Simplest Equation Method
}

\author{
Yun-Mei Zhao \\ Department of Mathematics, Honghe University, Mengzi, Yunnan 661100, China \\ Correspondence should be addressed to Yun-Mei Zhao; zhaoyunmei2000@126.com
}

Received 21 March 2014; Revised 8 June 2014; Accepted 9 June 2014; Published 29 June 2014

Academic Editor: Michael Meylan

Copyright ( 2014 Yun-Mei Zhao. This is an open access article distributed under the Creative Commons Attribution License, which permits unrestricted use, distribution, and reproduction in any medium, provided the original work is properly cited.

In our work, a generalized $\mathrm{KdV}$ type equation of neglecting the highest-order infinitesimal term, which is an important water wave model, is discussed by using the simplest equation method and its variants. The solutions obtained are general solutions which are in the form of hyperbolic, trigonometric, and rational functions. These methods are more effective and simple than other methods and a number of solutions can be obtained at the same time.

\section{Introduction}

In recent decades, the study of nonlinear partial differential equations (NLEEs) modelling physical phenomena has become an important research topic. Seeking exact solutions of NLEEs has long been one of the central themes of perpetual interest in mathematics and physics. With the development of symbolic computation packages like Maple and Mathematica, many powerful methods for finding exact solutions have been proposed, such as the homogeneous balance method [1], the extended $F$-expansion method [2], the auxiliary equation method [3], the sine-cosine method [4], the Jacobi elliptic function method [5], the exp-function method [6], the tanhfunction method [7], the $\left(G^{\prime} / G\right)$-expansion method [8], and the $\left(G^{\prime} / G, 1 / G\right)$-expansion method $[9,10]$.

The simplest equation method is a very powerful mathematical technique for finding exact solutions of nonlinear ordinary differential equations. It has been developed by Kudryashov [11, 12] and used successfully by many authors for finding exact solutions of ODEs in mathematical physics $[12,13]$.

Recently, Bilige et al. introduced a method called the extended simplest equation method, as an extension of the simplest equation method, to look for the exact traveling wave solutions of NLEEs [14, 15]. This method can construct different forms of exact traveling wave solutions which cannot be obtained by using the tanh-function method, $F$ expansion method, and the exp-function method.
In 1995, based on the physical and asymptotic considerations, Fokas [16] derived the following generalized KdV equation:

$$
\begin{aligned}
\eta_{t}+\eta_{x} & +\alpha \eta \eta_{x}+\beta \eta_{x x x}+\rho_{1} \alpha^{2} \eta^{2} \eta_{x} \\
& +\alpha \beta\left(\rho_{2} \eta \eta_{x x x}+\rho_{3} \eta_{x} \eta_{x x}\right)+\rho_{4} \alpha^{3} \eta^{3} \eta_{x} \\
& +\alpha^{2} \beta\left(\rho_{5} \eta^{2} \eta_{x x x}+\rho_{6} \eta \eta_{x} \eta_{x x}+\rho_{7} \eta_{x}^{3}\right)=0,
\end{aligned}
$$

which is an important water wave model, where $\alpha=3 A / 2$, $\beta=B / 6, \rho_{1}=-1 / 6, \rho_{2}=5 / 3, \rho_{3}=23 / 6, \rho_{4}=1 / 8, \rho_{5}=7 / 18$, $\rho_{6}=79 / 36$, and $\rho_{7}=45 / 36$. Regarding the $\rho_{1}, \rho_{2}, \rho_{3}, \rho_{4}, \rho_{5}$, $\rho_{6}$, and $\rho_{7}$ as free parameters and using the $\widetilde{\rho_{4}}$ to replace the $\rho_{4} \alpha^{2}$, (1) becomes the following PDE:

$$
\begin{aligned}
u_{t}+u_{x} & +\alpha u u_{x}+\beta u_{x x x}+\rho_{1} \alpha^{2} u^{2} u_{x} \\
& +\alpha \beta\left(\rho_{2} u u_{x x x}+\rho_{3} u_{x} u_{x x}\right)+\widetilde{\rho_{4}} \alpha u^{3} u_{x} \\
& +\alpha^{2} \beta\left(\rho_{5} u^{2} u_{x x x}+\rho_{6} u u_{x} u_{x x}+\rho_{7} u_{x}^{3}\right)=0,
\end{aligned}
$$

which is given by Tzirtzilakis et al. in [17]. They called it highorder wave equation of $\mathrm{KdV}$ type. Just as Tzirtzilakis et al. [17] said these two equations are both water wave equations of KdV type, which are more physically and practically meaningful. 
Assuming that the waves are unidirectional and neglecting terms of $O\left(\alpha^{2}, \alpha^{3}, \alpha \beta\right)$, (1) can be reduced to the classical $\mathrm{KdV}$ equation

$$
\eta_{t}+\eta_{x}+\alpha \eta \eta_{x}+\beta \eta_{x x x}=0
$$

Assuming that the $O(\beta)$ is less than $O(\alpha)$, this implies $O(\beta)<O(\alpha)$; then $O\left(\alpha^{2} \beta\right)<O\left(\alpha^{3}\right)$ and $O\left(\alpha^{2} \beta\right)<O(\alpha \beta)$. Neglecting two high-order infinitesimal terms of $O\left(\alpha^{3}, \alpha^{2} \beta\right)$, (1) can be reduced to another high-order wave equation of $\mathrm{KdV}$ type [17-20] as follows:

$$
\begin{aligned}
\eta_{t}+\eta_{x} & +\alpha \eta \eta_{x}+\beta \eta_{x x x}+\rho_{1} \alpha^{2} \eta^{2} \eta_{x} \\
& +\alpha \beta\left(\rho_{2} \eta \eta_{x x x}+\rho_{3} \eta_{x} \eta_{x x}\right)=0 .
\end{aligned}
$$

Equation (4) is a special case of (1) for $\rho_{4}=\rho_{5}=\rho_{6}=$ $\rho_{7}=0$.

If only we neglect the highest-order term of $\left(\alpha^{2} \beta\right)$, then (1) can be reduced to a new generalized $\mathrm{KdV}$ equation as follows:

$$
\begin{aligned}
\eta_{t}+\eta_{x} & +\alpha \eta \eta_{x}+\beta \eta_{x x x}+\rho_{1} \alpha^{2} \eta^{2} \eta_{x} \\
& +\alpha \beta\left(\rho_{2} \eta \eta_{x x x}+\rho_{3} \eta_{x} \eta_{x x}\right)+\rho_{4} \alpha^{3} \eta^{3} \eta_{x}=0 .
\end{aligned}
$$

In fact, (5) is another special case of (1) for $\rho_{5}=\rho_{6}=\rho_{7}=$ 0 . It is also third-order approximate equation of $\mathrm{KdV}$ type.

Of course, on describing dynamical behaviors of water waves, (4) is only a rough approximative model of (1) compared with (5); that is, the precision of model (5) is better than that of model (4) on describing dynamical behaviors of water waves. In other words, model (5) exhibits a much richer phenomenology than the model (4). Therefore, the investigation of exact traveling wave solutions for (5) is more practically meaningful than that of (4).

Equation (5) is studied by $\mathrm{Wu}$ et al. in [21] using the integral bifurcation method and some exact solutions in parameter form are given. In [22], some exact traveling wave solutions of (5) are given by using the extended $F$-expansion method [2]. In this paper, regarding the $\rho_{i}(i=1,2,3,4)$ as free parameters and by using the simplest equation method and its variants, we will investigate exact traveling wave solutions of (5).

The organization of the paper is as follows. In Section 2, a brief description of the simplest equation method and its variants for finding traveling wave solutions of nonlinear equations are given. In Section 3, we will study (5) by the simplest equation methods and its variants. Finally conclusions are given in Section 4 .

\section{Description of the Simplest Equation Method and Its Variants}

Consider a general nonlinear partial differential equation (PDE) for $u(x, t)$ in the form

$$
N\left(u, u_{t}, u_{x}, u_{t t}, u_{x t}, u_{x x}, \ldots\right)=0,
$$

where $N$ is a polynomial in its arguments.
By taking $u(x, t)=u(\xi)$ and $\xi=x-c t$, we look for traveling wave solutions of (6) and transform it to the ordinary differential equation (ODE)

$$
N\left(u,-c u^{\prime}, u^{\prime}, c^{2} u^{\prime \prime},-c u^{\prime \prime}, u^{\prime \prime}, \ldots\right)=0 .
$$

2.1. The Simplest Equation Method. Suppose the solution $u$ of (7) can be expressed as a finite series in the form

$$
u=\sum_{i=0}^{n} A_{i}(H(\xi))^{i},
$$

where $H(\xi)$ satisfies the Bernoulli or Riccati equation, $n$ is a positive integer that can be determined by balancing procedure, and $A_{i}(i=0,1,2, \ldots, n)$ are parameters to be determined.

The Bernoulli equation we consider in this paper is

$$
H^{\prime}(\xi)=a H(\xi)+b H^{2}(\xi),
$$

where $a$ and $b$ are constants. Its solutions can be written as

$$
\begin{aligned}
H(\xi) & =\frac{-a C_{1}}{b\left(C_{1}+\cosh \left(a\left(\xi+\xi_{0}\right)\right)-\sinh \left(a\left(\xi+\xi_{0}\right)\right)\right)}, \\
H(\xi) & =\frac{-a\left(\cosh \left(a\left(\xi+\xi_{0}\right)\right)+\sinh \left(a\left(\xi+\xi_{0}\right)\right)\right)}{b\left(C_{2}+\cosh \left(a\left(\xi+\xi_{0}\right)\right)+\sinh \left(a\left(\xi+\xi_{0}\right)\right)\right)},
\end{aligned}
$$

where $C_{1}, C_{2}$, and $\xi_{0}$ are constants.

For the Riccati equation

$$
H^{\prime}(\xi)=a H^{2}(\xi)+b H(\xi)+s,
$$

where $a, b$, and $s$ are constants. Equation (11) has 27 special solutions [23]; in this paper, we will use the following two solutions:

$$
\begin{aligned}
H(\xi)= & -\frac{b}{2 a}-\frac{\theta}{2 a} \tanh \left[\frac{\theta}{2}\left(\xi+\xi_{0}\right)\right], \\
H(\xi)= & -\frac{b}{2 a}-\frac{\theta}{2 a} \tanh \left(\frac{\theta}{2} \xi\right) \\
& +\frac{\operatorname{sech}(\theta \xi / 2)}{C \cosh (\theta \xi / 2)-(2 a / \theta) \sinh (\theta \xi / 2)},
\end{aligned}
$$

where $\theta^{2}=b^{2}-4 a s$

Substitute (8) into (7) with (9) (or (11)); then the lefthand side of (7) is converted into a polynomial in $H(\xi)$; equating each coefficient of the polynomial to zero yields a set of algebraic equations for $A_{i}, a, b(i=0,1,2, \ldots, n)$. Solving the algebraic equations by symbolic computation, we can determine those parameters explicitly.

Assuming that the constants $A_{i}, a, b(i=0,1,2, \ldots, n)$ can be obtained and substituting the results into (8), then we obtain the exact traveling wave solutions for (6).

Remark 1. In (9), when $a=A$ and $b=-1$ we obtain the Bernoulli equation

$$
H^{\prime}(\xi)=A H(\xi)-H^{2}(\xi) .
$$


Equation (13) admits the following exact solutions:

$$
H(\xi)=\frac{A}{2}\left(1+\tanh \left(\frac{A}{2}\left(\xi+\xi_{0}\right)\right)\right)
$$

when $A>0$, and

$$
H(\xi)=\frac{A}{2}\left(1-\tanh \left(\frac{A}{2}\left(\xi+\xi_{0}\right)\right)\right),
$$

when $A<0$.

2.2. The Generalized Simplest Equation Method. We Suppose the solution $u$ of (7) can be expressed in the following form:

$$
u=\sum_{i=0}^{n} a_{i} H^{i}+\sum_{j=1}^{n} b_{i} H^{-j}
$$

where $a_{i}, b_{j}(i=0,1,2, \ldots, n ; j=1,2, \ldots, n)$ are arbitrary constants to be determined later and $H=H(\xi)$ is

$$
H(\xi)=\frac{G^{\prime}}{G}
$$

where $G=G(\xi)$ satisfies the following auxiliary ordinary differential equation (ODE):

$$
A G G^{\prime \prime}-B G G^{\prime}-C\left(G^{\prime}\right)^{2}-E G^{2}=0,
$$

where the prime denotes derivative with respect to $\xi$. $A, B, C$, and $E$ are real parameters.

To determine the positive integer $n$, take the homogeneous balance between the highest-order nonlinear terms and the highest-order derivatives appearing in (7). Substituting (16) and (18) including (17) into (7) with the value of $n$ obtained and we obtain polynomials in $H^{n}(n=0,1,2, \ldots)$ and $H^{-n}(n=1,2, \ldots)$. Then, we collect each coefficient of the resulted polynomials to zero, yields a set of algebraic equations for $a_{i}(i=0,1,2, \ldots, n), b_{j}(j=1,2, \ldots, n)$, and $c$.

Suppose that the value of the constants $a_{i}(i=$ $0,1,2, \ldots, n), b_{j}(j=1,2, \ldots, n)$, and $c$ can be found by solving the algebraic equations which are obtained. Since the general solution of (18) is well known to us, substituting the values of $a_{i}(i=0,1,2, \ldots, n), b_{j}(j=1,2, \ldots, n)$, and $c$ into (16), we can obtain a more general type and new exact traveling wave solutions of the nonlinear partial differential equation (6).

The general solutions of (18) can be listed as follows:

(1) when $\Psi=A-C$ and $\Omega=B^{2}+4 E(A-C)>0$,

$$
\begin{aligned}
H(\xi)= & \frac{B}{2 \Psi}+\frac{\sqrt{\Omega}}{2 \Psi} \\
& \times \frac{C_{1} \sinh ((\sqrt{\Omega} / 2 A) \xi)+C_{2} \cosh ((\sqrt{\Omega} / 2 A) \xi)}{C_{1} \cosh ((\sqrt{\Omega} / 2 A) \xi)+C_{2} \sinh ((\sqrt{\Omega} / 2 A) \xi)},
\end{aligned}
$$

(2) when $\Psi=A-C$ and $\Omega=B^{2}+4 E(A-C)<0$,

$$
\begin{aligned}
H(\xi)= & \frac{B}{2 \Psi}+\frac{\sqrt{-\Omega}}{2 \Psi} \\
& \times \frac{-C_{1} \sin ((\sqrt{-\Omega} / 2 A) \xi)+C_{2} \cos ((\sqrt{-\Omega} / 2 A) \xi)}{C_{1} \cos ((\sqrt{-\Omega} / 2 A) \xi)+C_{2} \sin ((\sqrt{-\Omega} / 2 A) \xi)}
\end{aligned}
$$

(3) when $\Psi=A-C$ and $\Omega=B^{2}+4 E(A-C)=0$,

$$
H(\xi)=\frac{B}{2 \Psi}-\frac{A C_{2}}{\left(C_{1}-C_{2} \xi\right) \Psi} .
$$

2.3. The Extended Simplest Equation Method. We Suppose the solution $u$ of (7) can be expressed in the following form:

$$
u=\sum_{i=0}^{n} a_{i}\left(\frac{\phi^{\prime}}{\phi}\right)^{i}+\sum_{j=0}^{n-1} b_{i}\left(\frac{\phi^{\prime}}{\phi}\right)^{j} \frac{1}{\phi},
$$

where $a_{i}, b_{j}(i=0,1,2, \ldots, n ; j=0,1,2, \ldots, n-1)$ are constants and $a_{n} b_{n-1} \neq 0$. The positive number $n$ can be determined by considering the homogeneous balance between the highest-order derivatives and nonlinear terms appearing in (7). The function $\phi=\phi(\xi)$ satisfies the second order linear ODE in the form

$$
\phi^{\prime \prime}+\delta \phi=\mu,
$$

where $\delta$ and $\mu$ are constants. Equation (23) has three types of general solution with double arbitrary parameters as follows:

$$
\begin{aligned}
& \phi(\xi)=\left\{\begin{array}{cc}
A_{1} \cosh (\sqrt{-\delta} \xi) & \\
+A_{2} \sinh (\sqrt{-\delta} \xi)+\frac{\mu}{\delta}, & \text { when } \delta<0, \\
A_{1} \cos (\sqrt{\delta} \xi) & \text { when } \delta>0, \\
+A_{2} \sin (\sqrt{\delta} \xi)+\frac{\mu}{\delta}, & \text { when } \delta=0, \\
A_{1} \xi+A_{2}+\frac{\mu}{2} \xi^{2}, &
\end{array}\right. \\
& \left(\frac{\phi^{\prime}}{\phi}\right)^{2}= \begin{cases}\left(\delta A_{1}^{2}-\delta A_{2}^{2}-\frac{\mu^{2}}{\delta}\right)\left(\frac{1}{\phi}\right)^{2} & \text { when } \delta<0, \\
-\delta+\frac{2 \mu}{\phi}, & \text { when } \delta>0, \\
\left.-\delta A_{1}^{2}+\delta A_{2}^{2}-\frac{\mu^{2}}{\delta}\right)\left(\frac{1}{\phi}\right)^{2}, & \text { when } \delta=0, \\
\left(A_{1}^{2}-2 \mu A_{2}\right)\left(\frac{1}{\phi}\right)^{2}+\frac{2 \mu}{\phi}, & \end{cases}
\end{aligned}
$$

where $A_{1}$ and $A_{2}$ are arbitrary constants.

By substituting (22) into (7) and using the second order linear ODE (23) and (25), collecting all terms with the same order of $1 / \phi^{i}$ and $\left(1 / \phi^{i}\right)\left(\phi^{\prime} / \phi\right)$ together, the left-hand side of (7) is converted into another polynomial in $1 / \phi^{i}$ 
and $\left(1 / \phi^{i}\right)\left(\phi^{\prime} / \phi\right)$. Equating each coefficient of these different power terms to zero yields a set of algebraic equations for $a_{i}, b_{j}(i=0,1,2, \ldots, n ; j=0,1,2, \ldots, n-1), \delta$, and $\mu$.

Assume constants $a_{i}, b_{j}(i=0,1,2, \ldots, n ; j=$ $0,1,2, \ldots, n-1), \delta$, and $\mu$ can be determined by solving the nonlinear algebraic equations. Then substituting these terms and the general solutions (24) of (23) into (7), we can obtain more exact traveling wave solutions of (6).

\section{Exact Solutions of (5)}

Making a transformation $\eta(x, t)=u(\xi)$, with $\xi=x-c t,(5)$ can be reduced to the following ODE:

$$
\begin{aligned}
& (1-c) u^{\prime}+\alpha u u^{\prime}+\beta u^{\prime \prime \prime}+\rho_{1} \alpha^{2} u^{2} u^{\prime} \\
& +\alpha \beta\left(\rho_{2} u u^{\prime \prime \prime}+\rho_{3} u^{\prime} u^{\prime \prime}\right)+\rho_{4} \alpha^{3} u^{3} u^{\prime}=0
\end{aligned}
$$

where $c$ is wave velocity which moves along the direction of $x$-axis and $c \neq 0$. Integrating (11) once and setting the integral constant as $R$ yield

$$
\begin{aligned}
& (1-c) u+\frac{1}{2} \alpha u^{2}+\beta u^{\prime \prime}+\frac{1}{3} \rho_{1} \alpha^{2} u^{3} \\
& \quad+\alpha \beta\left(\rho_{2} u u^{\prime \prime}+\frac{1}{2}\left(\rho_{3}-\rho_{2}\right)+u^{\prime 2}\right)+\frac{1}{4} \rho_{4} \alpha^{3} u^{4}+R=0 .
\end{aligned}
$$

\subsection{Using the Simplest Equation Method}

3.1.1. Solutions of (27) Using the Bernoulli Equation as the Simplest Equation. Considering the homogeneous balance between $u u^{\prime \prime}$ and $u^{3}$, we get $n=1$, so the solution of (27) is in the form

$$
u(\xi)=A_{0}+A_{1} H(\xi) .
$$

Substituting (28) into (27) and making use of the Bernoulli equation (9) and then equating the coefficients of the functions $H^{i}(\xi)$ to zero, we obtain an algebraic system of equations in terms of $A_{i}(i=0,1), a, \rho_{i}(i=1,2,3,4)$, and $b$ as follows:

$$
\begin{aligned}
H(\xi)^{4}: & (1 / 4) \rho_{4} \alpha^{3} A_{1}^{4}+(3 / 2) \alpha \beta \rho_{2} A_{1}^{2} b^{2}+(1 / 2) \alpha \beta A_{1}^{2} \rho_{3} b^{2}=0, \\
H(\xi)^{3}: & \rho_{4} \alpha^{3} A_{0} A_{1}^{3}+2 \alpha \beta \rho_{2} A_{1} A_{0} b^{2}+2 \beta A_{1} b^{2}+\alpha \beta A_{1}^{2} \rho_{3} b a+ \\
& (1 / 3) \rho_{1} \alpha^{2} A_{1}^{3}+2 \alpha \beta \rho_{2} A_{1}^{2} b a=0 \\
H(\xi)^{2}: & (1 / 2) \alpha \beta A_{1}^{2} \rho_{3} a^{2}+(3 / 2) \rho_{4} \alpha^{3} A_{0}^{2} A_{1}^{2}+3 \alpha \beta \rho_{2} A_{1} A_{0} b a+ \\
& 3 \beta A_{1} b a+\rho_{1} \alpha^{2} A_{0} A_{1}^{2}+(1 / 2) \alpha A_{1}^{2}+(1 / 2) \alpha \beta \rho_{2} A_{1}^{2} a^{2}= \\
& 0, \\
H(\xi)^{1}: & \beta A_{1} a^{2}+A_{1}+\alpha A_{0} A_{1}+\alpha \beta \rho_{2} A_{1} A_{0} a^{2}+\rho_{4} \alpha^{3} A_{0}^{3} A_{1}+ \\
& \rho_{1} \alpha^{2} A_{0}^{2} A_{1}-c A_{1}=0, \\
H(\xi)^{0}: & -c A_{0}+(1 / 2) \alpha A_{0}^{2}+(1 / 3) \rho_{1} \alpha^{2} A_{0}^{3}+(1 / 4) \rho_{4} \alpha^{3} A_{0}^{4}+C+ \\
& A_{0}=0 .
\end{aligned}
$$

On solving the above algebraic equations using the Maple, we get the following results:

$$
\begin{gathered}
A_{0}=\frac{(3-\sqrt{3}) a A_{1}}{6 b}, \quad c=1+\frac{a^{4} \beta^{2} \rho_{3}}{3}, \\
\alpha=-\frac{2 \beta a b \sqrt{3}}{A_{1}}, \quad \rho_{1}=\frac{a^{2} \beta\left(\rho_{3}+2 \rho_{2}\right)-1}{2 a^{2} \beta}, \\
\rho_{4}=-\frac{3 \rho_{2}+\rho_{3}}{6 a^{2} \beta} .
\end{gathered}
$$

Therefore, using solutions (10) of (9), ansatz (28), we obtain the following exact solution of (5):

$$
\begin{aligned}
\eta_{1}(\xi)= & \frac{(3-\sqrt{3}) a A_{1}}{6 b} \\
& -\frac{A_{1} a C_{1}}{b\left(C_{1}+\cosh \left(a\left(\xi+\xi_{0}\right)\right)-\sinh \left(a\left(\xi+\xi_{0}\right)\right)\right)} \\
\eta_{2}(\xi)= & \frac{(3-\sqrt{3}) a A_{1}}{6 b} \\
& -\frac{A_{1} a\left(\cosh \left(a\left(\xi+\xi_{0}\right)\right)+\sinh \left(a\left(\xi+\xi_{0}\right)\right)\right)}{b\left(C_{2}+\cosh \left(a\left(\xi+\xi_{0}\right)\right)+\sinh \left(a\left(\xi+\xi_{0}\right)\right)\right)}
\end{aligned}
$$

where $\xi=x-\left(1+a^{4} \beta^{2} \rho_{3} / 3\right) t, \rho_{1}$ and $\rho_{4}$ are determined in (29), and $\rho_{2}, \rho_{3}, A_{1}, C_{1}, C_{2}, a$, and $b$ are arbitrary constants.

3.1.2. Solutions of (27) Using Riccati Equation as the Simplest Equation. Suppose the solution of (27) is of the form

$$
u(\xi)=B_{0}+B_{1} H(\xi)
$$

Substituting (32) into (27) and making use of the Riccati equation (11) and then equating the coefficients of the functions $H^{i}(\xi)$ to zero, we obtain an algebraic system of equations in terms of $B_{i}(i=0,1), a, b, \rho_{i}(i=1,2,3,4)$, and $s$ as follows:

$$
\begin{aligned}
H(\xi)^{4}: & (3 / 2) \alpha \beta \rho_{2} B_{1}^{2} a^{2}+(1 / 2) \alpha \beta B_{1}^{2} \rho_{3} a^{2}+(1 / 4) \rho_{4} \alpha^{3} B_{1}^{4}=0, \\
H(\xi)^{3}: & \alpha \beta B_{1}^{2} \rho_{3} a b+2 \alpha \beta \rho_{2} B_{1} B_{0} a^{2}+2 \beta B_{1} a^{2}+(1 / 3) \rho_{1} \alpha^{2} B_{1}^{3}+ \\
& \rho_{4} \alpha^{3} B_{0} B_{1}^{3}+2 \alpha \beta \rho_{2} B_{1}^{2} b a=0, \\
H(\xi)^{2}: & \rho_{1} \alpha^{2} B_{0} B_{1}^{2}+(1 / 2) \alpha \beta B_{1}^{2} \rho_{3} b^{2}+\alpha \beta \rho_{2} B_{1}^{2} a s+ \\
& (1 / 2) \alpha \beta \rho_{2} B_{1}^{2} b^{2}+(3 / 2) \rho_{4} \alpha^{3} B_{0}^{2} B_{1}^{2}+3 \alpha \beta \rho_{2} B_{1} B_{0} b a+ \\
& (1 / 2) \alpha B_{1}^{2}+3 \beta B_{1} a b+\alpha \beta B_{1}^{2} \rho_{3} a s=0, \\
H(\xi)^{1}: & \beta B_{1} b^{2}+\alpha \beta B_{1}^{2} \rho_{3} b s+B_{1}+\alpha \beta \rho_{2} B_{1} B_{0} b^{2}+\rho_{1} \alpha^{2} B_{0}^{2} B_{1}+ \\
& \alpha B_{0} B_{1}+2 \alpha \beta \rho_{2} B_{1} B_{0} a s+2 \beta B_{1} a s+\rho_{4} \alpha^{3} B_{0}^{3} B_{1}-c B_{1}=0, \\
H(\xi)^{0}: & -(1 / 2) \alpha \beta B_{1}^{2} \rho_{2} s^{2}+\beta B_{1} b s+(1 / 3) \rho_{1} \alpha^{2} B_{0}^{3}+ \\
& (1 / 4) \rho_{4} \alpha^{3} B_{0}^{4}+B_{0}+\alpha \beta \rho_{2} B_{1} B_{0} b s+(1 / 2) \alpha B_{0}^{2}+ \\
& C-c B_{0}+(1 / 2) \alpha \beta B_{1}^{2} \rho_{3} s^{2}=0 .
\end{aligned}
$$


On solving the above algebraic equations using the Maple, we get the following results:

$$
\begin{gathered}
B_{0}=\frac{(3 b+\sqrt{3 N}) B_{1}}{6 a}, \quad c=\frac{\beta^{2} \rho_{3} N^{2}}{3}+1, \\
\alpha=\frac{2 a \beta \sqrt{3 N}}{B_{1}}, \quad \rho_{1}=\frac{N \beta\left(\rho_{3}+2 \rho_{2}\right)-1}{2 \beta N}, \\
\rho_{4}=-\frac{3 \rho_{2}+\rho_{3}}{6 \beta N},
\end{gathered}
$$

where $N=b^{2}-4 a s$.

Therefore, using solutions (12) of (11), ansatz (32), we obtain the following exact solution of (5):

$$
\begin{gathered}
\eta_{3}(\xi)=\frac{(3 b+\sqrt{3 N}) B_{1}}{6 a}-\frac{B_{1}}{2 a}\left(b+\theta \tanh \left(\frac{\theta}{2}\left(\xi+\xi_{0}\right)\right)\right) \\
\eta_{4}(\xi)= \\
+\frac{(3 b+\sqrt{3 N}) B_{1}}{6 a}-\frac{B_{1}}{2 a}\left(b+\theta \tanh \left(\frac{\theta}{2} \xi\right)\right) \\
C \cosh ((\theta / 2) \xi)-(2 a / \theta) \sinh ((\theta / 2) \xi)
\end{gathered}
$$

where $\xi=x-\left(\beta^{2} \rho_{3} N^{2} / 3+1\right) t, \theta=\sqrt{b^{2}-4 a s}, N=b^{2}-4 a s$, $\rho_{1}$ and $\rho_{4}$ are determined in (33), and $\rho_{2}, \rho_{3}, B_{1}, C, s, a$, and $b$ are arbitrary constants.

3.2. Using Generalized Simplest Equation. Suppose the solution of (27) is of the form

$$
u(\xi)=a_{0}+a_{1} H(\xi)+\frac{b_{1}}{H(\xi)},
$$

where $a_{0}, a_{1}$, and $b_{1}$ are constants to be determined later and function $H=H(\xi)$ satisfies (17) and auxiliary differential equation (18).

Substituting (36) together with (17) and (18) into (27), the left-hand side is converted into polynomials in $H^{i},(i=$ $\ldots,-2,-1,0,1,2, \ldots)$. We collect each coefficient of these resulted polynomials to zero, yields a set of simultaneous algebraic equations for $a_{0}, a_{1}, b_{1}, \rho_{i}(i=1,2,3,4)$, and $c$. Solving this system of algebraic equations, with the aid of Maple, we obtain

$$
\begin{gathered}
a_{0}=\frac{b_{1}(\sqrt{3 M}+3 B)}{6 E}, \quad a_{1}=-\frac{b_{1} \Psi}{E}, \\
c=\frac{3 A^{4}+12 \beta^{2} E B \rho_{3} \Psi \sqrt{3 M}+\beta^{2} \rho_{3} M^{2}}{3 A^{4}}, \\
\rho_{1}=\frac{\beta\left(2 \rho_{2}+\rho_{3}\right) M-A^{2}}{2 \beta M}, \quad \rho_{4}=-\frac{A^{2}\left(3 \rho_{2}+\rho_{3}\right)}{6 \beta M}, \\
\alpha=\frac{2 \beta E \sqrt{3 M}}{b_{1} A^{2}},
\end{gathered}
$$

where $M=B^{2}-8 E \Psi$ and $\Psi=A-C . A, B, C, E, b_{1}, \rho_{2}, \rho_{3}$, and $\beta$ are free parameters.

Substituting (37) into (36), along with (19) and simplifying, yields the following traveling wave solutions (if $C_{1}=0$, but $C_{2} \neq 0 ; C_{2}=0$, but $\left.C_{1} \neq 0\right)$, respectively:

$$
\begin{aligned}
\eta_{5}(\xi)= & \frac{b_{1}(\sqrt{3 M}+3 B)}{6 E}-\frac{b_{1}}{2 E}\left(B+\sqrt{\Omega} \operatorname{coth}\left(\frac{\sqrt{\Omega}}{2 A} \xi\right)\right) \\
& +2 \Psi b_{1}\left(B+\sqrt{\Omega} \operatorname{coth}\left(\frac{\sqrt{\Omega}}{2 A} \xi\right)\right)^{-1},
\end{aligned}
$$

$$
\begin{aligned}
\eta_{6}(\xi)= & \frac{b_{1}(\sqrt{3 M}+3 B)}{6 E}-\frac{b_{1}}{2 E}\left(B+\sqrt{\Omega} \tanh \left(\frac{\sqrt{\Omega}}{2 A} \xi\right)\right) \\
& +2 \Psi b_{1}\left(B+\sqrt{\Omega} \tanh \left(\frac{\sqrt{\Omega}}{2 A} \xi\right)\right)^{-1},
\end{aligned}
$$

where $\xi=x-c t, M=B^{2}-8 E \Psi, \Psi=A-C, \Omega=B^{2}+4 E \Psi, c$, $\rho_{1}$, and $\rho_{4}$ are determined in (37), and $\rho_{2}, \rho_{3}, A, B, C, E$, and $b_{1}$, are arbitrary constants.

Substituting (37) into (36), along with (20) and simplifying, yields the following traveling wave solutions (if $C_{1}=0$, but $C_{2} \neq 0 ; C_{2}=0$, but $\left.C_{1} \neq 0\right)$, respectively:

$$
\begin{aligned}
\eta_{7}(\xi)= & \frac{b_{1}(\sqrt{3 M}+3 B)}{6 E}-\frac{b_{1}}{2 E}\left(B+\sqrt{-\Omega} \cot \left(\frac{\sqrt{-\Omega}}{2 A} \xi\right)\right) \\
& +2 \Psi b_{1}\left(B+\sqrt{-\Omega} \cot \left(\frac{\sqrt{-\Omega}}{2 A} \xi\right)\right)^{-1}, \\
\eta_{8}(\xi)= & \frac{b_{1}(\sqrt{3 M}+3 B)}{6 E}-\frac{b_{1}}{2 E}\left(B-\sqrt{-\Omega} \tan \left(\frac{\sqrt{-\Omega}}{2 A} \xi\right)\right) \\
& +2 \Psi b_{1}\left(B-\sqrt{-\Omega} \tan \left(\frac{\sqrt{-\Omega}}{2 A} \xi\right)\right)^{-1},
\end{aligned}
$$

where $\xi=x-c t, M=B^{2}-8 E \Psi, \Psi=A-C, \Omega=B^{2}+4 E \Psi$, $c, \rho_{1}$, and $\rho_{4}$ are determined in (37), and $\rho_{2}, \rho_{3}, A, B, C, E$, and $b_{1}$, are arbitrary constants.

Substituting (37) into (36), along with (21), and simplifying, our obtained solution becomes

$$
\eta_{9}(\xi)=-\frac{8 C_{2}^{2} A^{2} b_{1} \Psi}{\left(2 A C_{2}+B C_{2} \xi-B C_{1}\right)\left(C_{1}-C_{2} \xi\right) B^{2}},
$$

where $\xi=x-c t, \Psi=A-C, \rho_{1}$ and $\rho_{4}$ are determined in (37), and $\rho_{2}, \rho_{3}, A, B, C_{1}, C_{2}$, and $b_{1}$ are arbitrary constants. 
3.3. Using Extended Simplest Equation. Suppose the solution of (27) is of the form

$$
u(\xi)=a_{0}+a_{1} \frac{\phi^{\prime}}{\phi}+b_{1} \frac{1}{\phi}
$$

where $a_{0}, a_{1}$, and $b_{1}$ are constants to be determined later and function $\phi=\phi(\xi)$ satisfies the second order linear ODE (23).

By substituting (42) into (27) and using the second order linear ODE expressions (23) and (25), collecting all terms with the same order of $1 / \phi^{i}$ and $\left(1 / \phi^{i}\right)\left(\phi^{\prime} / \phi\right)$ together, the lefthand side of (27) is converted into another polynomial in $1 / \phi^{i}$ and $\left(1 / \phi^{i}\right)\left(\phi^{\prime} / \phi\right)$. Equating each coefficient of these different power terms to zero yields a set of algebraic equations for $a_{0}$, $a_{1}, b_{1}, \rho_{1}, \rho_{2}, \rho_{3}, \rho_{4}, \alpha, \beta, c, \delta$, and $\mu$. Solving these equations, we obtain the following results.

If $\delta<0$, we obtain

(1) $b_{1}=0$,

$$
\begin{aligned}
& c=-\frac{2 \alpha \beta a_{0}^{3} \rho_{3}-6 \beta a_{0}^{2}+2 \alpha \beta a_{0} \rho_{3} a_{1}^{2} \delta-2 \beta a_{1}^{2} \delta-a_{1}^{2}}{a_{1}^{2}}, \\
& \mu=0, \\
& \rho_{1}=\frac{6\left(3 a_{0}^{2} \beta\left(3-a_{0} \alpha \rho_{3}\right)-a_{1}^{2}\left(a_{0} \alpha+\beta \delta+a_{0} \alpha \beta \rho_{3} \delta\right)\right)}{\alpha^{2} a_{1}^{2}\left(3 a_{0}^{2}+a_{1}^{2} \delta\right)}, \\
& \rho_{2}=\frac{6 a_{0} \beta\left(2-a_{0} \alpha \rho_{3}\right)-\alpha a_{1}^{2}\left(2 \beta \rho_{3} \delta+1\right)}{2 \alpha \beta\left(3 a_{0}^{2}+a_{1}^{2} \delta\right)} \\
& \rho_{4}=\frac{\alpha a_{1}^{2}\left(4 \beta \rho_{3} \delta+3\right)+12 a_{0} \beta\left(a_{0} \alpha \rho_{3}-3\right)}{\alpha^{3} a_{1}^{2}\left(3 a_{0}^{2}+a_{1}^{2} \delta\right)} .
\end{aligned}
$$

(2) $a_{0}=\sqrt{\frac{-\delta}{3}} a_{1}, \quad b_{1}=0, \quad c=1+\frac{16 \beta^{2} \rho_{3} \delta^{2}}{3}$,

$$
\begin{aligned}
& \mu=0, \quad \alpha=\frac{4 \sqrt{-3 \delta} \beta}{a_{1}}, \\
& \rho_{1}=\frac{1+8 \beta \rho_{2} \delta+4 \delta \beta \rho_{3}}{8 \delta \beta}, \\
& \rho_{4}=\frac{3 \rho_{2}+\rho_{3}}{24 \beta \delta} .
\end{aligned}
$$

(3) $A_{1}= \pm A_{2}, \quad a_{0}=-\frac{1}{\alpha \rho_{2}}, \quad b_{1}=0$,

$$
c=\frac{-2-a_{1}^{2} \delta \alpha^{2} \rho_{2}^{2}+\rho_{2} M}{\rho_{2} M}, \quad \beta=-\frac{\alpha^{2} a_{1}^{2} \rho_{2}}{M}
$$$$
\rho_{1}=\frac{6 \rho_{2}}{M}, \quad \rho_{3}=-2 \rho_{2} \text {, }
$$$$
\rho_{4}=\frac{2 \rho_{2}^{2}}{M}, \quad M=6+a_{1}^{2} \delta \alpha^{2} \rho_{2}^{2} .
$$

(4) $a_{0}=-\frac{1}{\alpha \rho_{2}}, \quad b_{1}=0$,

$$
\begin{aligned}
& c=\frac{-2 a_{1}^{2} \delta \alpha^{2} \rho_{2}^{2}-1+\rho_{2} N}{\rho_{2} N}, \\
& \mu=\sqrt{A_{1}^{2}-A_{2}^{2}} \delta, \quad \beta=\frac{\alpha^{2} a_{1}^{2} \rho_{2}}{N}, \quad \rho_{1}=\frac{3 \rho_{2}}{N}, \\
& \rho_{3}=-5 \rho_{2}, \quad \rho_{4}=\frac{\rho_{2}^{2}}{N}, \quad N=3+2 a_{1}^{2} \delta \alpha^{2} \rho_{2}^{2} .
\end{aligned}
$$

(5) $A_{1}=\frac{\sqrt{\mu^{2}+\delta^{2} A_{2}^{2}}}{\delta}, \quad a_{0}=-\frac{\delta \beta}{\alpha}, \quad a_{1}=\frac{\sqrt{-3 \delta} \beta}{\alpha}$,

$$
b_{1}=0, \quad c=2 \rho_{4} \delta^{3} \beta^{3}+1-\delta^{2} \beta^{2} \rho_{2},
$$$$
\rho_{1}=\frac{1-\beta \rho_{2} \delta+6 \delta^{2} \rho_{4} \beta^{2}}{2 \beta \delta}, \quad \rho_{3}=-3 \rho_{2}+6 \delta \beta \rho_{4} \text {. }
$$

(6) $A_{1}= \pm A_{2}, \quad a_{0}=-\frac{\delta \beta}{\alpha}, \quad a_{1}=\frac{\sqrt{-3 \delta} \beta}{\alpha}$,

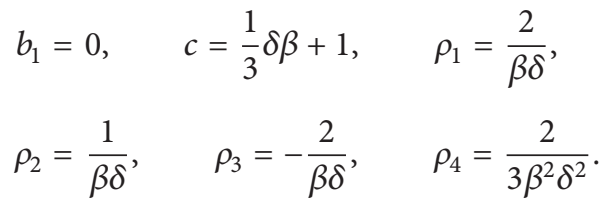

(7) $A_{1}=\frac{\sqrt{9 A_{2}^{2} \beta^{2} \delta^{2}-3 b_{1}^{2} \alpha^{2}}}{3 \delta \beta}, \quad a_{0}=-\frac{\delta \beta}{\alpha}$, $a_{1}=\frac{\sqrt{-3 \delta} \beta}{\alpha}, \quad c=2 \rho_{4} \delta^{3} \beta^{3}-\beta \delta+1, \quad \mu=0$, $\rho_{1}=3 \delta \beta \rho_{4}, \quad \rho_{2}=\frac{1}{\beta \delta}, \quad \rho_{3}=\frac{3\left(2 \delta^{2} \rho_{4} \beta^{2}-1\right)}{\beta \delta}$.

(8) $a_{0}=\frac{b_{1} \sqrt{3\left(A_{2}^{2}-A_{1}^{2}\right)}}{3\left(A_{1}^{2}-A_{2}^{2}\right)}, \quad a_{1}=\frac{b_{1}}{\sqrt{\delta\left(A_{1}^{2}-A_{2}^{2}\right)}}$, $c=2 \rho_{4} \delta^{3} \beta^{3}+1-\delta^{2} \beta^{2} \rho_{2}, \quad \mu=0$,

$$
\begin{aligned}
\alpha & =\frac{\sqrt{3\left(A_{2}^{2}-A_{1}^{2}\right)} \delta \beta}{b_{1}}, \quad \rho_{1}=\frac{1-\beta \rho_{2} \delta+6 \delta^{2} \rho_{4} \beta^{2}}{2 \beta \delta}, \\
\rho_{3} & =6 \delta \beta \rho_{4}-3 \rho_{2} .
\end{aligned}
$$

(9) $A_{1}= \pm A_{2}, \quad a_{0}=\frac{b_{1} \delta}{\mu}, \quad a_{1}=\frac{\sqrt{-\delta} b_{1}}{\mu}$, $c=1-\beta \delta, \quad \rho_{1}=-\frac{3 \mu\left(\alpha \beta \rho_{2} b_{1} \delta+2 \beta \mu+\alpha b_{1}\right)}{2 b_{1}^{2} \delta \alpha^{2}}$, 


$$
\begin{aligned}
& \rho_{3}=\frac{3 \beta \mu+\alpha b_{1}-\alpha \beta \rho_{2} b_{1} \delta}{b_{1} \delta \alpha \beta}, \\
& \rho_{4}=\frac{\mu^{2}\left(2 \alpha \beta \rho_{2} b_{1} \delta+3 \beta \mu+\alpha b_{1}\right)}{2 b_{1}^{3} \delta^{2} \alpha^{3}} .
\end{aligned}
$$

(10) $A_{1}=\frac{\sqrt{\delta^{2} A_{2}^{2}-2 \mu^{2}}}{\delta}, \quad a_{0}=\frac{b_{1} \delta}{3 \mu}$,

$$
\begin{aligned}
& a_{1}=\frac{\sqrt{-3 \delta} b_{1}}{3 \mu}, \\
& c=\frac{27 \mu^{3}-2 \rho_{4} \alpha^{3} b_{1}^{3} \delta^{3}-3 b_{1}^{2} \delta^{2} \mu \alpha^{2} \rho_{2}}{27 \mu^{3}}, \\
& \beta=-\frac{b_{1} \alpha}{3 \mu}, \\
& \rho_{1}=-\frac{\alpha \rho_{2} \mu b_{1} \delta+3 \mu^{2}+2 b_{1}^{2} \delta^{2} \rho_{4} \alpha^{2}}{2 \mu b_{1} \delta \alpha}, \\
& \rho_{3}=-\frac{3 \mu \rho_{2}+2 \rho_{4} \alpha b_{1} \delta}{\mu} .
\end{aligned}
$$

Substituting (43)-(52) into (42) and making use of solutions (24) of (23), we can obtain, respectively, exact traveling wave solutions expressed by hyperbolic functions of (5). Consider

$$
\eta_{10}=a_{0}+\frac{a_{1} \sqrt{-\delta}\left(A_{1} \sinh (\sqrt{-\delta} \xi)+A_{2} \cosh (\sqrt{-\delta} \xi)\right)}{A_{1} \cosh (\sqrt{-\delta} \xi)+A_{2} \sinh (\sqrt{-\delta} \xi)},
$$

where $\xi=x+\left(\left(2 \alpha \beta a_{0}^{3} \rho_{3}-6 \beta a_{0}^{2}+2 \alpha \beta a_{0} \rho_{3} a_{1}^{2} \delta-2 \beta a_{1}^{2} \delta-\right.\right.$ $\left.\left.a_{1}^{2}\right) / a_{1}^{2}\right) t, \rho_{1}, \rho_{2}$, and $\rho_{4}$ are determined in (43), and $\alpha, \beta, \rho_{3}$, $a_{0}, a_{1}, A_{1}, A_{2}$, and $\delta$ are arbitrary constants. Consider

$$
\begin{aligned}
\eta_{11}= & \frac{\sqrt{-3 \delta}}{3} a_{1} \\
& +\frac{a_{1} \sqrt{-\delta}\left(A_{1} \sinh (\sqrt{-\delta} \xi)+A_{2} \cosh (\sqrt{-\delta} \xi)\right)}{A_{1} \cosh (\sqrt{-\delta} \xi)+A_{2} \sinh (\sqrt{-\delta} \xi)},
\end{aligned}
$$

where $\xi=x-\left(1+16 \beta^{2} \rho_{3} \delta^{2} / 3\right) t, \alpha, \rho_{1}$, and $\rho_{4}$ are determined in (44), and $\beta, \rho_{2}, \rho_{3}, a_{1}, A_{1}, A_{2}$, and $\delta$ are arbitrary constants. Consider

$$
\begin{aligned}
\eta_{12}= & -\frac{1}{\alpha \rho_{2}} \\
& +\frac{a_{1} \sqrt{-\delta}\left( \pm A_{2} \sinh (\sqrt{-\delta} \xi)+A_{2} \cosh (\sqrt{-\delta} \xi)\right)}{ \pm A_{2} \cosh (\sqrt{-\delta} \xi)+A_{2} \sinh (\sqrt{-\delta} \xi)+\mu / \delta}
\end{aligned}
$$

where $\xi=x-\left(\left(-2-a_{1}^{2} \delta \alpha^{2} \rho_{2}^{2}+\rho_{2}\left(6+a_{1}^{2} \delta \alpha^{2} \rho_{2}^{2}\right)\right) / \rho_{2}(6+\right.$ $\left.\left.a_{1}^{2} \delta \alpha^{2} \rho_{2}^{2}\right)\right) t, \beta, \rho_{1}, \rho_{3}$, and $\rho_{4}$ are determined in (45), and $\alpha$, $a_{1}, A_{2}, \rho_{2}, \mu$, and $\delta$ are arbitrary constants. Consider

$$
\begin{aligned}
\eta_{13}= & -\frac{1}{\alpha \rho_{2}} \\
& +\frac{a_{1} \sqrt{-\delta}\left(A_{1} \sinh (\sqrt{-\delta} \xi)+A_{2} \cosh (\sqrt{-\delta} \xi)\right)}{A_{1} \cosh (\sqrt{-\delta} \xi)+A_{2} \sinh (\sqrt{-\delta} \xi)+\sqrt{A_{1}^{2}-A_{2}^{2}}},
\end{aligned}
$$

where $\xi=x-\left(\left(-2 a_{1}^{2} \delta \alpha^{2} \rho_{2}^{2}-1+\rho_{2}\left(3+2 a_{1}^{2} \delta \alpha^{2} \rho_{2}^{2}\right)\right) / \rho_{2}(3+\right.$ $\left.\left.2 a_{1}^{2} \delta \alpha^{2} \rho_{2}^{2}\right)\right) t, \beta, \rho_{1}, \rho_{3}$, and $\rho_{4}$ are determined in (46), and $\alpha$, $a_{1}, A_{1}, A_{2}, \rho_{2}$, and $\delta$ are arbitrary constants. Consider

$$
\begin{aligned}
\eta_{14}= & -\frac{\delta \beta}{\alpha} \\
& \times\left(1+\frac{\sqrt{3}\left(A_{1} \sinh (\sqrt{-\delta} \xi)+A_{2} \cosh (\sqrt{-\delta} \xi)\right)}{A_{1} \cosh (\sqrt{-\delta} \xi)+A_{2} \sinh (\sqrt{-\delta} \xi)+\mu / \delta}\right),
\end{aligned}
$$

where $\xi=x-\left(2 \rho_{4} \delta^{3} \beta^{3}+1-\delta^{2} \beta^{2} \rho_{2}\right) t, A_{1}, \rho_{1}$, and $\rho_{3}$ are determined in (47), and $\alpha, \beta, A_{2}, \rho_{2}, \rho_{4}, \mu$, and $\delta$ are arbitrary constants. Consider

$$
\begin{aligned}
\eta_{15}= & -\frac{\delta \beta}{\alpha} \\
& \times\left(1-\frac{\sqrt{3}\left( \pm A_{2} \sinh (\sqrt{-\delta} \xi)+A_{2} \cosh (\sqrt{-\delta} \xi)\right)}{ \pm A_{2} \cosh (\sqrt{-\delta} \xi)+A_{2} \sinh (\sqrt{-\delta} \xi)+\mu / \delta}\right),
\end{aligned}
$$

where $\xi=x-((1 / 3) \delta \beta+1) t, \rho_{1}, \rho_{2}, \rho_{3}$, and $\rho_{4}$ are determined in (48), and $\alpha, \beta, A_{2}, \mu$, and $\delta$ are arbitrary constants. Consider

$$
\begin{aligned}
\eta_{16}= & -\frac{\delta \beta}{\alpha}\left(1+\frac{\sqrt{3}\left(A_{1} \sinh (\sqrt{-\delta} \xi)+A_{2} \cosh (\sqrt{-\delta} \xi)\right)}{A_{1} \cosh (\sqrt{-\delta} \xi)+A_{2} \sinh (\sqrt{-\delta} \xi)}\right) \\
& +\frac{b_{1}}{A_{1} \cosh (\sqrt{-\delta} \xi)+A_{2} \sinh (\sqrt{-\delta} \xi)}
\end{aligned}
$$

where $\xi=x-\left(2 \rho_{4} \delta^{3} \beta^{3}-\beta \delta+1\right) t, A_{1}, \rho_{1}, \rho_{2}$, and $\rho_{3}$ are determined in (49), and $\alpha, \beta, b_{1}, A_{2}, \rho_{4}$, and $\delta$ are arbitrary constants. Consider

$\eta_{17}$

$$
\begin{aligned}
= & \frac{b_{1} \sqrt{3\left(A_{2}^{2}-A_{1}^{2}\right)}}{3\left(A_{1}^{2}-A_{2}^{2}\right)} \\
& +\frac{\left(b_{1} / \sqrt{A_{2}^{2}-A_{1}^{2}}\right)\left(A_{1} \sinh (\sqrt{-\delta} \xi)+A_{2} \cosh (\sqrt{-\delta} \xi)\right)+b_{1}}{A_{1} \cosh (\sqrt{-\delta} \xi)+A_{2} \sinh (\sqrt{-\delta} \xi)},
\end{aligned}
$$


where $\xi=x-\left(2 \rho_{4} \delta^{3} \beta^{3}+1-\delta^{2} \beta^{2} \rho_{2}\right) t, \alpha, \rho_{1}$, and $\rho_{3}$ are determined in (50), and $\beta, b_{1}, A_{1}, A_{2}, \rho_{2}, \rho_{4}$, and $\delta$ are arbitrary constants. Consider

$$
\begin{aligned}
\eta_{18}= & \frac{b_{1} \delta}{\mu} \\
& \times\left(1-\frac{ \pm A_{2} \sinh (\sqrt{-\delta} \xi)+A_{2} \cosh (\sqrt{-\delta} \xi)}{ \pm A_{2} \cosh (\sqrt{-\delta} \xi)+A_{2} \sinh (\sqrt{-\delta} \xi)+\mu / \delta}\right) \\
& +\frac{b_{1}}{ \pm A_{2} \cosh (\sqrt{-\delta} \xi)+A_{2} \sinh (\sqrt{-\delta} \xi)+\mu / \delta},
\end{aligned}
$$

where $\xi=x-(1-\beta \delta) t, \rho_{1}, \rho_{3}$, and $\rho_{4}$ are determined in (51), and $\alpha, \beta, b_{1}, A_{2}, \rho_{2}, \mu$, and $\delta$ are arbitrary constants. Consider

$$
\begin{aligned}
\eta_{19}= & \frac{b_{1} \delta}{3 \mu} \\
& \times\left(1-\frac{\sqrt{3}\left(A_{1} \sinh (\sqrt{-\delta} \xi)+A_{2} \cosh (\sqrt{-\delta} \xi)\right)}{A_{1} \cosh (\sqrt{-\delta} \xi)+A_{2} \sinh (\sqrt{-\delta} \xi)+\mu / \delta}\right) \\
& +\frac{b_{1}}{A_{1} \cosh (\sqrt{-\delta} \xi)+A_{2} \sinh (\sqrt{-\delta} \xi)+\mu / \delta},
\end{aligned}
$$

where $\xi=x-\left(\left(27 \mu^{3}-2 \rho_{4} \alpha^{3} b_{1}^{3} \delta^{3}-3 b_{1}^{2} \delta^{2} \mu \alpha^{2} \rho_{2}\right) / 27 \mu^{3}\right) t, A_{1}$, $\beta, \rho_{1}$, and $\rho_{3}$ are determined in (52), and $\alpha, b_{1}, A_{2}, \rho_{2}, \rho_{4}, \mu$, and $\delta$ are arbitrary constants.

If $\delta>0$, we obtain

(1) $a_{0}=i a_{1} \sqrt{\frac{\delta}{3}}, \quad b_{1}=0, \quad c=1+\frac{16 \beta^{2} \rho_{3} \delta^{2}}{3}$,

$$
\begin{aligned}
& \alpha=\frac{4 \beta i \sqrt{3 \delta}}{a_{1}}, \quad \rho_{1}=\frac{1+8 \beta \rho_{2} \delta+4 \delta \beta \rho_{3}}{8 \delta \beta}, \\
& \mu=0, \quad \rho_{4}=\frac{3 \rho_{2}+\rho_{3}}{24 \beta \delta} .
\end{aligned}
$$

(2) $A_{1}=i A_{2}, \quad a_{0}=-\frac{1}{\alpha \rho_{2}}, \quad b_{1}=0$,

$$
\begin{aligned}
c & =\frac{-2-a_{1}^{2} \delta \alpha^{2} \rho_{2}^{2}+\rho_{2} M}{\rho_{2} M}, \quad \beta=-\frac{\alpha^{2} a_{1}^{2} \rho_{2}}{M}, \\
\rho_{1} & =\frac{6 \rho_{2}}{M}, \quad \rho_{3}=-2 \rho_{2}, \\
\rho_{4} & =\frac{2 \rho_{2}^{2}}{M}, \quad M=6+a_{1}^{2} \delta \alpha^{2} \rho_{2}^{2} .
\end{aligned}
$$

(3) $A_{1}=\frac{\sqrt{\mu^{2}-\delta^{2} A_{2}^{2}}}{\delta}, \quad a_{0}=-\frac{\delta \beta}{\alpha}, \quad a_{1}=\frac{\sqrt{3 \delta} \beta i}{\alpha}$,

$$
b_{1}=0, \quad c=2 \rho_{4} \delta^{3} \beta^{3}+1-\delta^{2} \beta^{2} \rho_{2},
$$$$
\rho_{1}=\frac{1-\beta \rho_{2} \delta+6 \delta^{2} \rho_{4} \beta^{2}}{2 \beta \delta}, \quad \rho_{3}=-3 \rho_{2}+6 \delta \beta \rho_{4} \text {. }
$$

(4) $A_{1}=i A_{2}, \quad a_{0}=-\frac{\delta \beta}{\alpha}, \quad a_{1}=\frac{\sqrt{3 \delta} \beta i}{\alpha}$,

$$
b_{1}=0, \quad c=\frac{1}{3} \delta \beta+1, \quad \rho_{1}=\frac{2}{\beta \delta},
$$

$$
\rho_{2}=\frac{1}{\beta \delta}, \quad \rho_{3}=-\frac{2}{\beta \delta}, \quad \rho_{4}=\frac{2}{3 \beta^{2} \delta^{2}} .
$$

(5) $A_{1}=\frac{\sqrt{-9 A_{2}^{2} \beta^{2} \delta^{2}-3 b_{1}^{2} \alpha^{2}}}{3 \delta \beta}, \quad a_{0}=-\frac{\delta \beta}{\alpha}$,

$$
\begin{aligned}
& a_{1}=\frac{\sqrt{3 \delta} \beta i}{\alpha}, \quad c=2 \rho_{4} \delta^{3} \beta^{3}-\delta^{2} \beta^{2} \rho_{2}+1, \\
& \mu=0, \quad \rho_{1}=\frac{1-\beta \rho_{2} \delta+6 \delta^{2} \rho_{4} \beta^{2}}{2 \beta \delta} \\
& \rho_{3}=-3 \rho_{2}+6 \delta \beta \rho_{4} .
\end{aligned}
$$

(6) $A_{1}=i A_{2}, \quad a_{0}=\frac{b_{1} \delta}{\mu}, \quad a_{1}=\frac{\sqrt{\delta} b_{1} i}{\mu}$,

$$
\begin{aligned}
c & =1-\beta \delta, \quad \rho_{1}=-\frac{3 \mu\left(\alpha \beta \rho_{2} b_{1} \delta+2 \beta \mu+\alpha b_{1}\right)}{2 b_{1}^{2} \delta \alpha^{2}}, \\
\rho_{3} & =\frac{3 \beta \mu+\alpha b_{1}-\alpha \beta \rho_{2} b_{1} \delta}{b_{1} \delta \alpha \beta}, \\
\rho_{4} & =\frac{\mu^{2}\left(2 \alpha \beta \rho_{2} b_{1} \delta+3 \beta \mu+\alpha b_{1}\right)}{2 b_{1}^{3} \delta^{2} \alpha^{3}} .
\end{aligned}
$$

(7) $A_{1}=\frac{\sqrt{-\delta^{2} A_{2}^{2}-2 \mu^{2}}}{\delta}, \quad a_{0}=\frac{b_{1} \delta}{3 \mu}$,

$$
\begin{aligned}
& a_{1}=\frac{i \sqrt{3 \delta} b_{1}}{3 \mu}, \\
& c=\frac{27 \mu^{3}-2 \rho_{4} \alpha^{3} b_{1}^{3} \delta^{3}-3 b_{1}^{2} \delta^{2} \mu \alpha^{2} \rho_{2}}{27 \mu^{3}}, \quad \beta=-\frac{b_{1} \alpha}{3 \mu},
\end{aligned}
$$$$
\rho_{1}=-\frac{\alpha \rho_{2} \mu b_{1} \delta+3 \mu^{2}+2 b_{1}^{2} \delta^{2} \rho_{4} \alpha^{2}}{2 \mu b_{1} \delta \alpha}
$$$$
\rho_{3}=-\frac{3 \mu \rho_{2}+2 \rho_{4} \alpha b_{1} \delta}{\mu} .
$$ 
Substituting (63)-(69) into (42) and making use of solutions (24) of (23), we can obtain, respectively, exact traveling wave solutions expressed by trigonometric functions of (5). Consider

$$
\eta_{20}=\frac{\sqrt{3 \delta} a_{1} i}{3}+\frac{a_{1} \sqrt{\delta}\left(-A_{1} \sin (\sqrt{\delta} \xi)+A_{2} \cos (\sqrt{\delta} \xi)\right)}{A_{1} \cos (\sqrt{\delta} \xi)+A_{2} \sin (\sqrt{\delta} \xi)},
$$

where $\xi=x-\left(1+16 \beta^{2} \rho_{3} \delta^{2} / 3\right) t, \alpha, \rho_{1}$, and $\rho_{4}$ are determined in (63), and $\beta, \rho_{2}, \rho_{3}, a_{1}, A_{1}, A_{2}$, and $\delta$ are arbitrary constants. Consider

$$
\eta_{21}=-\frac{1}{\alpha \rho_{2}}+\frac{a_{1} \sqrt{\delta}\left(-i A_{2} \sin (\sqrt{\delta} \xi)+A_{2} \cos (\sqrt{\delta} \xi)\right)}{i A_{2} \cos (\sqrt{\delta} \xi)+A_{2} \sin (\sqrt{\delta} \xi)+\mu / \delta},
$$

where $\xi=x-\left(\left(-2-a_{1}^{2} \delta \alpha^{2} \rho_{2}^{2}+\rho_{2}\left(6+a_{1}^{2} \delta \alpha^{2} \rho_{2}^{2}\right)\right) / \rho_{2}(6+\right.$ $\left.\left.a_{1}^{2} \delta \alpha^{2} \rho_{2}^{2}\right)\right) t, \beta, \rho_{1}, \rho_{3}$, and $\rho_{4}$ are determined in (64), and $\alpha$, $a_{1}, A_{2}, \rho_{2}, \mu$, and $\delta$ are arbitrary constants. Consider

$$
\eta_{22}=-\frac{\delta \beta}{\alpha}\left(1-\frac{\sqrt{3} i\left(-A_{1} \sin (\sqrt{\delta} \xi)+A_{2} \cos (\sqrt{\delta} \xi)\right)}{A_{1} \cos (\sqrt{\delta} \xi)+A_{2} \sin (\sqrt{\delta} \xi)+\mu / \delta}\right),
$$

where $\xi=x-\left(2 \rho_{4} \delta^{3} \beta^{3}+1-\delta^{2} \beta^{2} \rho_{2}\right) t, A_{1}, \rho_{1}$, and $\rho_{3}$ are determined in (65), and $\alpha, \beta, A_{2}, \rho_{2}, \rho_{4}, \mu$, and $\delta$ are arbitrary constants. Consider

$$
\eta_{23}=-\frac{\delta \beta}{\alpha}\left(1-\frac{\sqrt{3} i\left(-i A_{2} \sin (\sqrt{\delta} \xi)+A_{2} \cos (\sqrt{\delta} \xi)\right)}{i A_{2} \cos (\sqrt{\delta} \xi)+A_{2} \sin (\sqrt{\delta} \xi)+\mu / \delta}\right),
$$

where $\xi=x-((1 / 3) \delta \beta+1) t, \rho_{1}, \rho_{2}, \rho_{3}$, and $\rho_{4}$ are determined in (66), and $\alpha, \beta, A_{2}, \mu$, and $\delta$ are arbitrary constants. Consider

$$
\begin{aligned}
\eta_{24}= & -\frac{\delta \beta}{\alpha}\left(1-\frac{\sqrt{3} i\left(-A_{1} \sin (\sqrt{\delta} \xi)+A_{2} \cos (\sqrt{\delta} \xi)\right)}{A_{1} \cos (\sqrt{\delta} \xi)+A_{2} \sin (\sqrt{\delta} \xi)}\right) \\
& +\frac{b_{1}}{A_{1} \cos (\sqrt{\delta} \xi)+A_{2} \sin (\sqrt{\delta} \xi)},
\end{aligned}
$$

where $\xi=x-\left(2 \rho_{4} \delta^{3} \beta^{3}-\delta^{2} \beta^{2} \rho_{2}+1\right) t, A_{1}, \rho_{1}$, and $\rho_{3}$ are determined in (67), and $\alpha, \beta, b_{1}, A_{2}, \rho_{2}, \rho_{4}$, and $\delta$ are arbitrary constants. Consider

$$
\begin{aligned}
\eta_{25}= & \frac{b_{1} \delta}{\mu}\left(1+\frac{i\left(-i A_{2} \sin (\sqrt{\delta} \xi)+A_{2} \cos (\sqrt{\delta} \xi)\right)}{i A_{2} \cos (\sqrt{\delta} \xi)+A_{2} \sin (\sqrt{\delta} \xi)+\mu / \delta}\right) \\
& +\frac{b_{1}}{i A_{2} \cos (\sqrt{\delta} \xi)+A_{2} \sin (\sqrt{\delta} \xi)+\mu / \delta},
\end{aligned}
$$

where $\xi=x-(1-\beta \delta) t, \rho_{1}, \rho_{3}$, and $\rho_{4}$ are determined in (68), and $\alpha, \beta, b_{1}, A_{2}, \rho_{2}, \mu$, and $\delta$ are arbitrary constants. Consider

$$
\begin{aligned}
\eta_{26}= & \frac{b_{1} \delta}{3 \mu}\left(1+\frac{\sqrt{3} i\left(-A_{1} \sin (\sqrt{\delta} \xi)+A_{2} \cos (\sqrt{\delta} \xi)\right)}{A_{1} \cos (\sqrt{\delta} \xi)+A_{2} \sin (\sqrt{\delta} \xi)+\mu / \delta}\right) \\
& +\frac{b_{1}}{A_{1} \cos (\sqrt{\delta} \xi)+A_{2} \sin (\sqrt{\delta} \xi)+\mu / \delta},
\end{aligned}
$$

where $\xi=x-\left(\left(27 \mu^{3}-2 \rho_{4} \alpha^{3} b_{1}^{3} \delta^{3}-3 b_{1}^{2} \delta^{2} \mu \alpha^{2} \rho_{2}\right) / 27 \mu^{3}\right) t, A_{1}$, $\beta, \rho_{1}$, and $\rho_{3}$ are determined in (69), and $\alpha, b_{1}, A_{2}, \rho_{2}, \rho_{4}, \mu$, and $\delta$ are arbitrary constants.

If $\delta=0$, we obtain

(1) $a_{0}=0, \quad a_{1}=0, \quad c=1, \quad \alpha=-\frac{6 \beta \mu}{b_{1}}$,

$$
\rho_{1}=\frac{3 \beta \rho_{3} \mu^{2}+2 \mu A_{2}+6 \beta \rho_{2} \mu^{2}-A_{1}^{2}}{6 \beta \mu^{2}}
$$

$$
\rho_{4}=\frac{6 \rho_{2} \mu A_{2}-3 \rho_{2} A_{1}^{2}-\rho_{3} A_{1}^{2}+2 \rho_{3} \mu A_{2}}{18 \beta \mu^{2}} .
$$

(2) $A_{2}=\frac{a_{0} A_{1}^{2}-\mu b_{1}}{2 a_{0} \mu}, \quad a_{1}=0, \quad c=\frac{6 \beta^{2} a_{0}^{2} \mu^{2} \rho_{3}+b_{1}^{2}}{b_{1}^{2}}$,

$$
\alpha=\frac{6 \beta \mu}{b_{1}}, \quad \rho_{1}=\frac{6 \beta \rho_{2} a_{0} \mu+3 \beta \mu \rho_{3} a_{0}-b_{1}}{6 \mu \beta a_{0}},
$$

$\rho_{4}=-\frac{b_{1}\left(3 \rho_{2}+\rho_{3}\right)}{18 \mu \beta a_{0}}$.

(3) $b_{1}=0, \quad c=\frac{6 a_{0}^{2} \beta-2 a_{0}^{3} \alpha \beta \rho_{3}+a_{1}^{2}}{a_{1}^{2}}, \quad \mu=0$,

$\rho_{1}=\frac{2\left(9 a_{0} \beta-3 \alpha a_{0}^{2} \beta \rho_{3}-\alpha a_{1}^{2}\right)}{a_{0} \alpha^{2} a_{1}^{2}}$,

$\rho_{2}=\frac{12 a_{0} \beta-6 \alpha a_{0}^{2} \beta \rho_{3}-\alpha a_{1}^{2}}{6 \alpha a_{0}^{2} \beta}$

$\rho_{4}=\frac{4 \alpha a_{0}^{2} \beta \rho_{3}-12 a_{0} \beta+\alpha a_{1}^{2}}{\alpha^{3} a_{0}^{2} a_{1}^{2}}$.

(4) $A_{1}=\frac{b_{1}}{a_{1}}, \quad c=\frac{1}{2} \alpha a_{0}\left(1-\rho_{4} \alpha^{2} a_{0}^{2}\right)+1$,

$\mu=0, \quad \rho_{1}=-\frac{3 \rho_{4} \alpha^{2} a_{0}^{2}+1}{2 \alpha a_{0}}$,

$\rho_{2}=-\frac{3 \rho_{4} \alpha^{3} a_{0}^{2} a_{1}^{2}+3 a_{0} \beta-\alpha a_{1}^{2}}{3 \alpha a_{0}^{2} \beta}$,

$\rho_{3}=\frac{\rho_{4} \alpha^{3} a_{0}^{2} a_{1}^{2}+3 a_{0} \beta-\alpha a_{1}^{2}}{\alpha a_{0}^{2} \beta}$. 


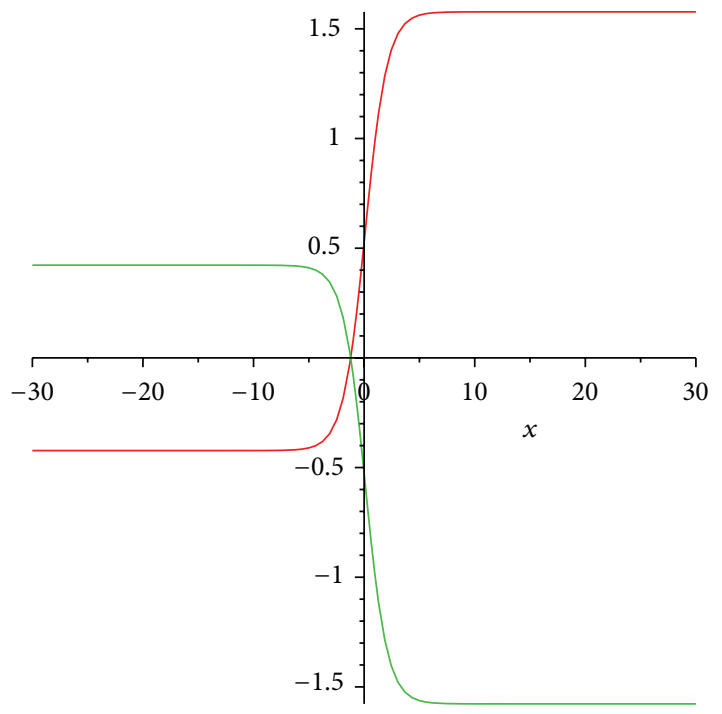

(a) $A_{1}=2,-2, t=0.1$

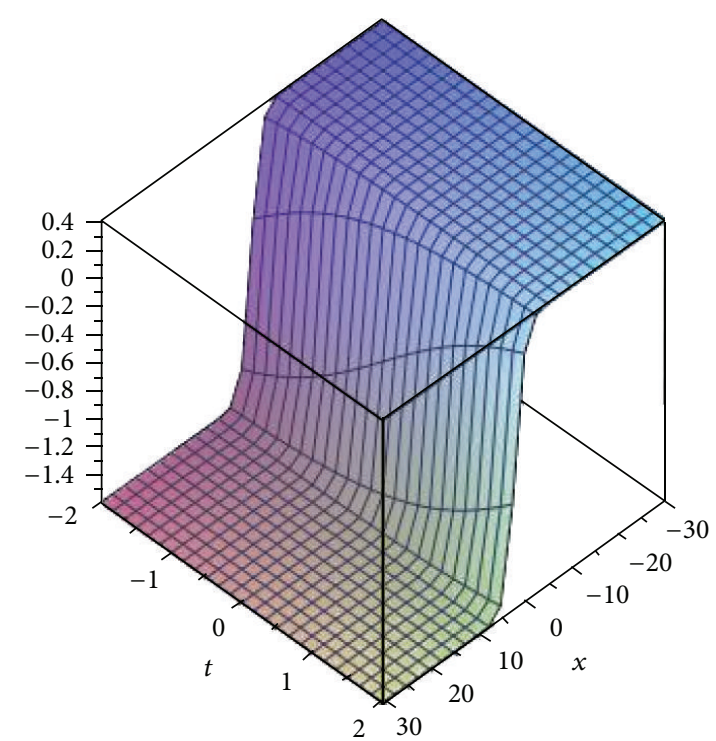

(b) $A_{1}=2,-2 \leq t \leq 2$

Figure 1: 2D and 3D figures of solution $\eta_{1}(\xi)$ in (30) with $a=1, b=1, C_{1}=1, \xi_{0}=0, \rho_{3}=0.1, \alpha=0.1$, and $\beta=0.2$ in the intervals $x \in[-30,30]$.

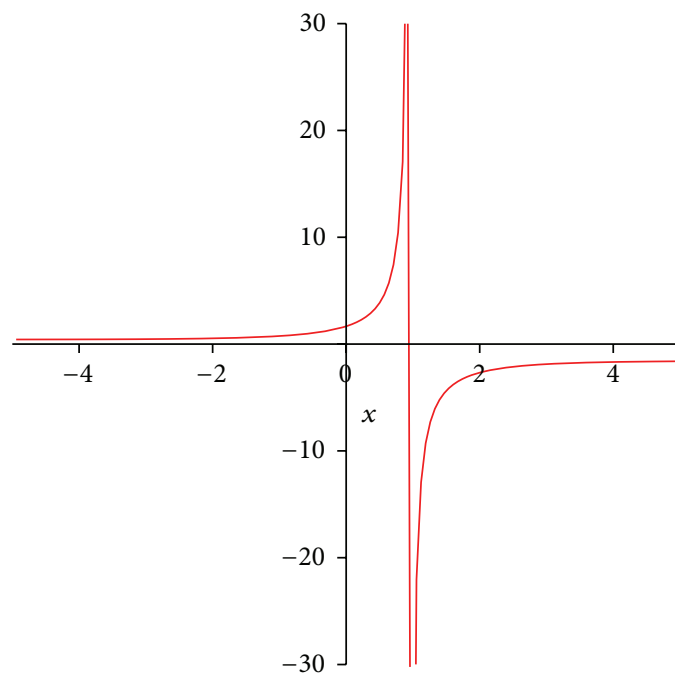

(a) $t=0.2$

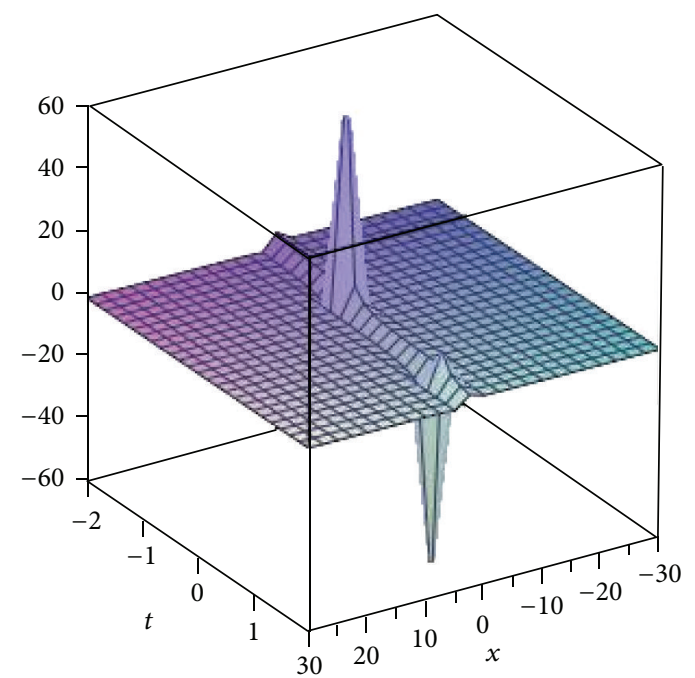

(b) $-2 \leq t \leq 2$

Figure 2: 2D and 3D figures of solution $\eta_{2}(\xi)$ in (31) with $a=1, b=1, A_{1}=2, C_{2}=-2, \xi_{0}=0, \rho_{3}=1, \alpha=1$, and $\beta=1$ in the intervals $x \in[-30,30]$.

(5) $A_{2}=\frac{A_{1}^{2}}{2 \mu}, \quad b_{1}=0, \quad c=\frac{1}{2} \alpha a_{0}\left(1-\rho_{4} \alpha^{2} a_{0}^{2}\right)+1, \quad \rho_{3}=\frac{\rho_{4} \alpha^{3} a_{0}^{2} a_{1}^{2}+3 a_{0} \beta-\alpha a_{1}^{2}}{\alpha a_{0}^{2} \beta}$.

$$
\begin{array}{ll}
\rho_{1}=-\frac{3 \rho_{4} \alpha^{2} a_{0}^{2}+1}{2 \alpha a_{0}}, & \text { (6) } c=\frac{3 a_{0}^{2} \beta+3 a_{0}^{3} \alpha \beta \rho_{2}+2 \alpha a_{0} a_{1}^{2}+6 a_{1}^{2}}{6 a_{1}^{2}}, \\
\rho_{2}=-\frac{3 \rho_{4} \alpha^{3} a_{0}^{2} a_{1}^{2}+3 a_{0} \beta-\alpha a_{1}^{2}}{3 \alpha a_{0}^{2} \beta}, & \rho_{1}=\frac{3 a_{0} \beta+3 \alpha a_{0}^{2} \beta \rho_{2}-2 \alpha a_{1}^{2}}{2 a_{0} \alpha^{2} a_{1}^{2}},
\end{array}
$$




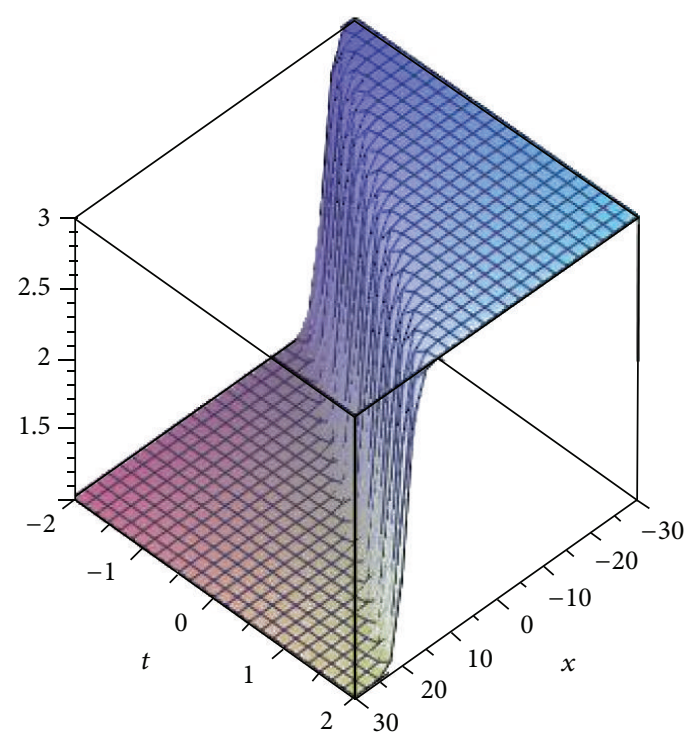

(a) $\eta_{3}$

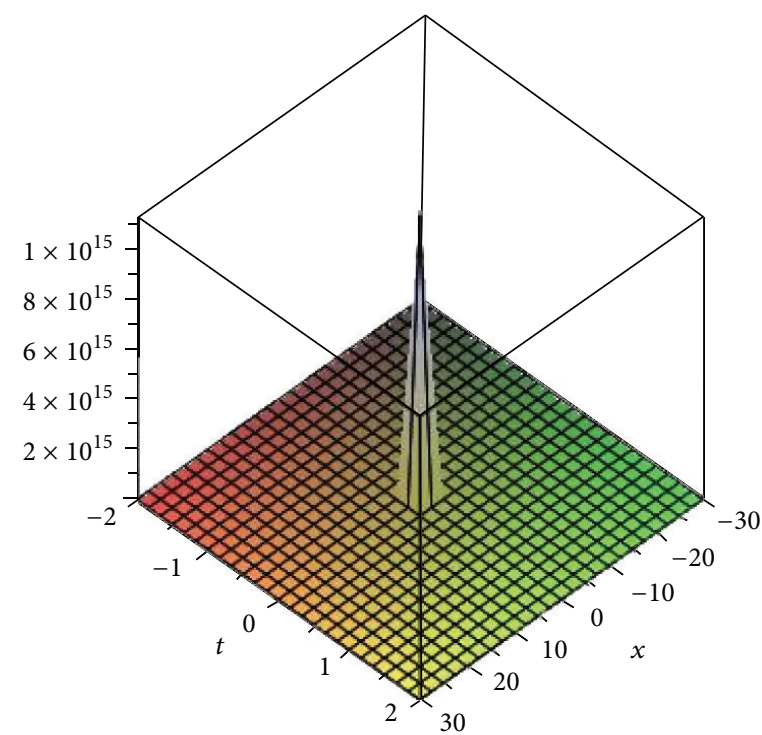

(b) $\eta_{4}$

Figure 3: 3D figures of solution $\eta_{3}(\xi)$ in (34) and $\eta_{4}(\xi)$ in (35) with $a=-1, b=1, B_{1}=-2, N=12, \xi_{0}=2, \theta=1, \rho_{3}=5$, and $\beta=0.2$ in the intervals $x \in[-30,30], t \in[-2,2]$.

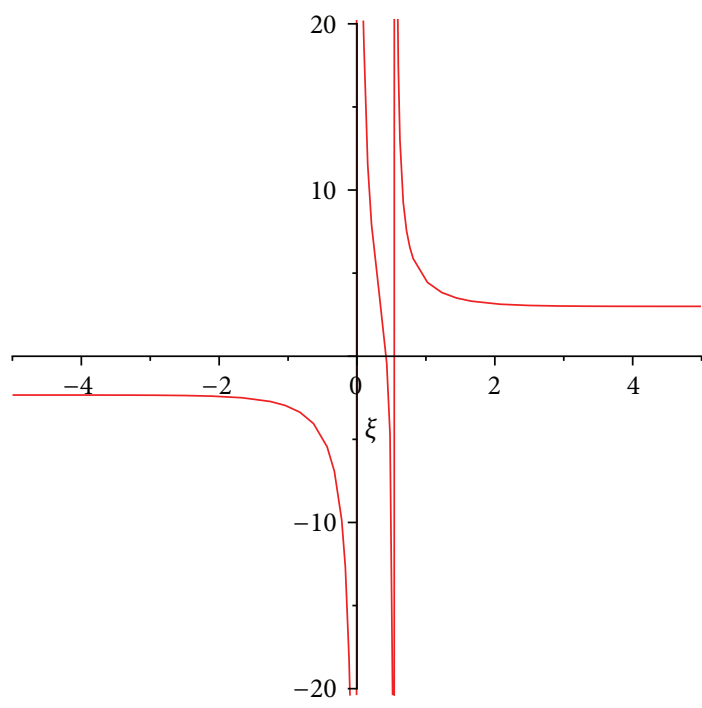

(a) $\eta_{5}, b_{1}=2,-2$

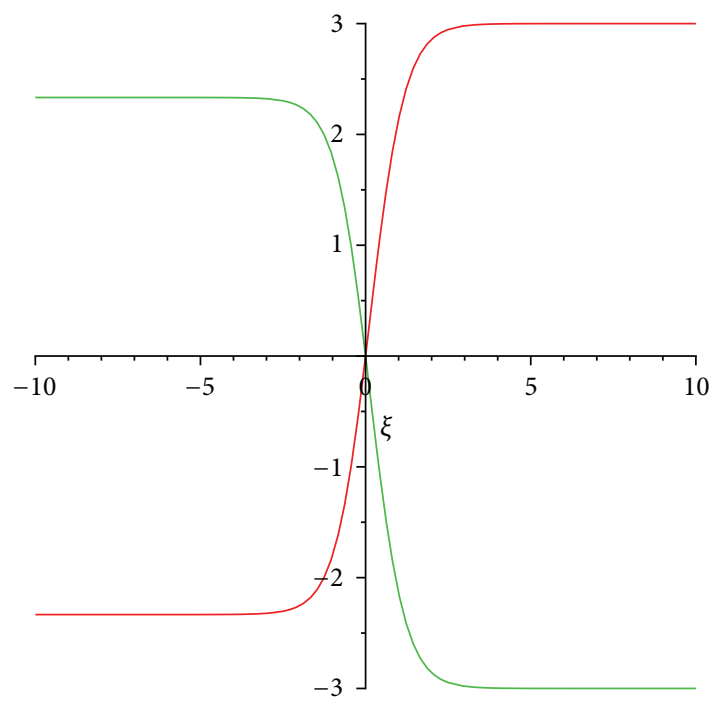

(b) $\eta_{6}, b_{1}=-2$

Figure 4: $2 \mathrm{D}$ figures of solution $\eta_{5}(\xi)$ in (38) and $\eta_{6}(\xi)$ in (39) with $a=-1, B=-4, \Omega=4, M=3, E=1, \Psi=1$, and $A=1$ in the intervals $\xi \in[-10,10]$.

$$
\begin{aligned}
\rho_{3} & =\frac{6 a_{0} \beta-3 \alpha a_{0}^{2} \beta \rho_{2}-2 \alpha a_{1}^{2}}{3 \alpha a_{0}^{2} \beta}, \\
\rho_{4} & =-\frac{3 a_{0} \beta+3 \alpha a_{0}^{2} \beta \rho_{2}-\alpha a_{1}^{2}}{3 \alpha^{3} a_{0}^{2} a_{1}^{2}}, \\
A_{2} & =\frac{a_{1}^{2} A_{1}^{2}-b_{1}^{2}}{2 \mu a_{1}^{2}} .
\end{aligned}
$$

Substituting (77)-(82) into (42) and making use of solutions (24) of (23), we can obtain, respectively, exact traveling wave solutions expressed by rational functions of (5). Consider

$$
\eta_{27}=\frac{2 b_{1}}{2 A_{1} \xi+2 A_{2}+\mu \xi^{2}},
$$




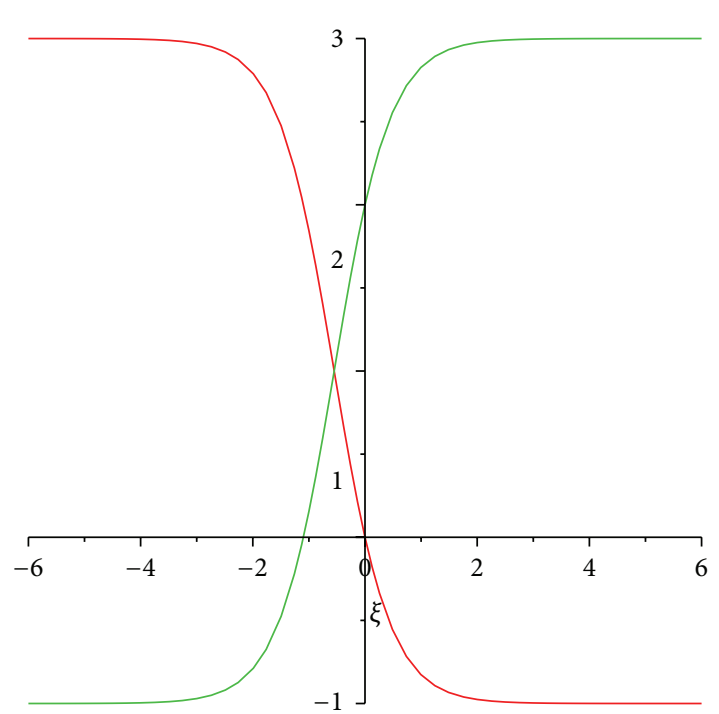

(a) $\eta_{10}, a_{1}=2,-2,-6 \leq \xi \leq 6$

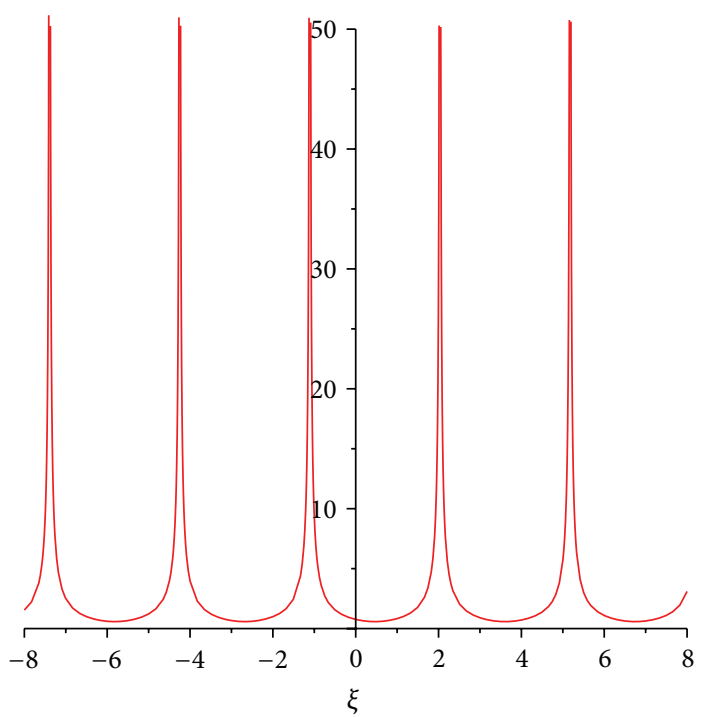

(b) $\eta_{20},-8 \leq \xi \leq 8$

Figure 5: 2D figures of solution $\eta_{10}(\xi)$ in (53) with $a_{0}=1, A_{1}=2, A_{2}=1$, and $\delta=-1$ and $\eta_{20}(\xi)$ in $(70)$ with $a_{1}=1, A_{1}=2, A_{2}=1$, and $\delta=1$.

where $\xi=x-t, \alpha, \rho_{1}$, and $\rho_{4}$ are determined in (77), and $\beta$, $b_{1}, A_{1}, A_{2}, \rho_{2}, \rho_{3}$, and $\mu$ are arbitrary constants. Consider

$$
\eta_{28}=a_{0}+\frac{2 b_{1}}{2 A_{1} \xi+\left(a_{0} A_{1}^{2}-\mu b_{1}\right) / a_{0} \mu+\mu \xi^{2}},
$$

where $\xi=x-\left(\left(6 \beta^{2} a_{0}^{2} \mu^{2} \rho_{3}+b_{1}^{2}\right) / b_{1}^{2}\right) t, \alpha, \rho_{1}$, and $\rho_{4}$ are determined in (78), and $\beta, a_{0}, b_{1}, A_{1}, \rho_{2}, \rho_{3}$, and $\mu$ are arbitrary constants.Consider

$$
\eta_{29}=a_{0}+\frac{a_{1} A_{1}}{A_{1} \xi+A_{2}},
$$

where $\xi=x-\left(\left(6 a_{0}^{2} \beta-2 a_{0}^{3} \alpha \beta \rho_{3}+a_{1}^{2}\right) / a_{1}^{2}\right) t, \rho_{1}, \rho_{2}$, and $\rho_{4}$ are determined in (79), and $\alpha, \beta, a_{0}, a_{1}, A_{1}, A_{2}$, and $\rho_{3}$ are arbitrary constants. Consider

$$
\eta_{30}=a_{0}+\frac{2 b_{1} a_{1}}{b_{1} \xi+A_{2} a_{1}},
$$

where $\xi=x-\left((1 / 2) \alpha a_{0}\left(1-\rho_{4} \alpha^{2} a_{0}^{2}\right)+1\right) t, \rho_{1}, \rho_{2}$, and $\rho_{3}$ are determined in (80), and $\alpha, \beta, a_{0}, a_{1}, b_{1}, A_{2}$, and $\rho_{4}$ are arbitrary constants. Consider

$$
\eta_{31}=a_{0}+\frac{2 \mu a_{1}\left(A_{1}+\mu \xi\right)}{2 \mu A_{1} \xi+A_{1}^{2}+\mu^{2} \xi^{2}},
$$

where $\xi=x-\left((1 / 2) \alpha a_{0}\left(1-\rho_{4} \alpha^{2} a_{0}^{2}\right)+1\right) t, \rho_{1}, \rho_{2}$, and $\rho_{3}$ are determined in (81), and $\alpha, \beta, a_{0}, a_{1}, A_{1}, \rho_{4}$, and $\mu$ are arbitrary constants. Consider

$$
\eta_{32}=a_{0}+\frac{2 \mu a_{1}^{2}\left(a_{1}\left(A_{1}+\mu \xi\right)+b_{1}\right)}{2 \mu a_{1}^{2} A_{1} \xi-b_{1}^{2}+a_{1}^{2} A_{1}^{2}+a_{1}^{2} \mu^{2} \xi^{2}},
$$

where $\xi=x-\left(\left(3 a_{0}^{2} \beta+3 a_{0}^{3} \alpha \beta \rho_{2}+2 \alpha a_{0} a_{1}^{2}+6 a_{1}^{2}\right) / 6 a_{1}^{2}\right) t, \rho_{1}, \rho_{3}$, and $\rho_{4}$ are determined in (82), and $\alpha, \beta, a_{0}, a_{1}, b_{1}, A_{1}, \rho_{2}$, and $\mu$ are arbitrary constants.

\section{Figures of Some Exact Solutions}

In this section, some typical wave figures are given as in Figures 1, 2, 3, 4, and 5.

\section{Conclusions}

In this paper, with the aid of Maple, we successfully obtained wider classes of exact traveling solutions of (5) by using the simplest equation method and its variants. And some new exact solutions expressed by hyperbolic function, trigonometric function, and rational functions are obtained and some typical wave figures are given including periodic wave, solitary wave, kink wave, and some new types. The simplest equation method and its variants can construct different forms of exact traveling wave solutions which cannot be obtained by using the tanh-function method, $F$-expansion method, $\left(G^{\prime} / G\right)$-expansion method, and the exp-function method. The correctness of all the solutions is verified by substituting them into original equation (5). Comparing with $[21,22]$, it is easy to see that our method is more straightforward and the form of the solutions obtained in our paper is also more simple and many solutions are new. The related results are enriched.

\section{Conflict of Interests}

The author declares that there is no conflict of interests regarding the publication of this paper.

\section{Acknowledgments}

This work was supported by the National Natural Science Foundation of China (11161020; 11361023), the Natural Science Foundation of Yunnan Province (2011FZ193), and the Natural 
Science Foundation of Education Committee of Yunnan Province (2012Y452 and 2013C079).

\section{References}

[1] M. Wang, "Exact solutions for a compound KdV-Burgers equation," Physics Letters. A, vol. 213, no. 5-6, pp. 279-287, 1996.

[2] E. Yomba, "The extended Fan's sub-equation method and its application to $\mathrm{KdV}-\mathrm{MKdV}, \mathrm{BKK}$ and variant Boussinesq equations," Physics Letters A, vol. 336, no. 6, pp. 463-476, 2005.

[3] Sirendaoreji and S. Jiong, "Auxiliary equation method for solving nonlinear partial differential equations," Physics Letters A, vol. 309, no. 5-6, pp. 387-396, 2003.

[4] Z. Yan and H. Zhang, "New explicit and exact travelling wave solutions for a system of variant Boussinesq equations in mathematical physics," Physics Letters A, vol. 252, no. 6, pp. 291296, 1999.

[5] E. Fan and J. Zhang, "Applications of the Jacobi elliptic function method to special-type nonlinear equations," Physics Letters. A, vol. 305, no. 6, pp. 383-392, 2002.

[6] X. Wu and J. He, "EXP-function method and its application to nonlinear equations," Chaos, Solitons \& Fractals, vol. 38, no. 3, pp. 903-910, 2008.

[7] H. A. Abdusalam, "On an improved complex tanh-function method," International Journal of Nonlinear Sciences and Numerical Simulation, vol. 6, no. 2, pp. 99-106, 2005.

[8] M. Wang, X. Li, and J. Zhang, "The $G^{\prime} / G$-expansion method and travelling wave solutions of nonlinear evolution equations in mathematical physics," Physics Letters. A, vol. 372, no. 4, pp. 417-423, 2008.

[9] E. M. E. Zayed and S. A. Hoda Ibrahim, "The two variable $\left(G^{\prime} / G, 1 / G\right)$-expansion method for finding exact traveling wave solutions of the (3+1)-dimensional nonlinear potential YuToda-Sasa-Fukuyama equation," in Proceedings of the International Conference on Advanced Computer Science and Electronics Information, pp. 388-392, 2013.

[10] E. M. E. Zayed and S. A. Hoda Ibrahim, "The $\left(G^{\prime} / G, 1 / G\right)$ expansion method and its applications for constructing the exact solutions of the nonlinear Zoomeron equation," Transaction on IoT and Cloud Computing, vol. 2, no. 1, pp. 66-75, 2014.

[11] N. A. Kudryashov, "Exact solitary waves of the Fisher equation," Physics Letters A, vol. 342, no. 1-2, pp. 99-106, 2005.

[12] N. A. Kudryashov, "Simplest equation method to look for exact solutions of nonlinear differential equations," Chaos, Solitons and Fractals, vol. 24, no. 5, pp. 1217-1231, 2005.

[13] N. K. Vitanov and Z. I. Dimitrova, "Application of the method of simplest equation for obtaining exact traveling-wave solutions for two classes of model PDEs from ecology and population dynamics," Communications in Nonlinear Science and Numerical Simulation, vol. 15, no. 10, pp. 2836-2845, 2010.

[14] S. Bilige and T. Chaolu, "An extended simplest equation method and its application to several forms of the fifth-order $\mathrm{KdV}$ equation," Applied Mathematics and Computation, vol. 216, no. 11, pp. 3146-3153, 2010.

[15] S. Bilige, T. Chaolu, and X. Wang, "Application of the extended simplest equation method to the coupled SchrödingerBoussinesq equation," Applied Mathematics and Computation, vol. 224, pp. 517-523, 2013.

[16] A. S. Fokas, "On a class of physically important integrable equations," Physica D: Nonlinear Phenomena, vol. 87, no. 1-4, pp. 145-150, 1995.
[17] E. Tzirtzilakis, V. Marinakis, C. Apokis, and T. Bountis, "Soliton-like solutions of higher order wave equations of the Korteweg-de Vries type," Journal of Mathematical Physics, vol. 43, no. 12, pp. 6151-6165, 2002.

[18] E. Tzirtzilakis, M. Xenos, V. Marinakis, and T. C. Bountis, "Interactions and stability of solitary waves in shallow water," Chaos, Solitons \& Fractals, vol. 14, no. 1, pp. 87-95, 2002.

[19] J. Li, W. Rui, Y. Long, and B. He, "Travelling wave solutions for higher-order wave equations of KdV type (III)," Mathematical Biosciences and Engineering, vol. 3, no. 1, pp. 125-135, 2006.

[20] W. G. Rui, Y. Long, and B. He, "Some new travelling wave solutions with singular or nonsingular character for the higher order wave equation of KdV type (III)," Nonlinear Analysis: Theory, Methods \& Applications, vol. 70, no. 11, pp. 3816-3828, 2009.

[21] X. Wu, W. Rui, and X. Hong, "A generalized KdV equation of neglecting the highest-order infinitesimal term and its exact traveling wave solutions," Abstract and Applied Analysis, vol. 2013, Article ID 656297, 15 pages, 2013.

[22] Y. He, Y. Zhao, and Y. Long, "New exact solutions for a higher-order wave equation of $\mathrm{KdV}$ type using extended Fexpansion method," Mathematical Problems in Engineering, vol. 2013, Article ID 128970, 8 pages, 2013.

[23] H. Naher and F. A. Abdullah, "New traveling wave solutions by the extended generalized Riccati equation mapping method of the $(2+1)$-dimensional evolution equation," Journal of Applied Mathematics, vol. 2012, Article ID 486458, 18 pages, 2012. 


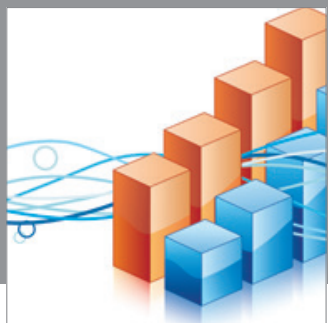

Advances in

Operations Research

mansans

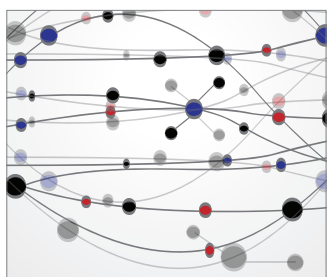

The Scientific World Journal
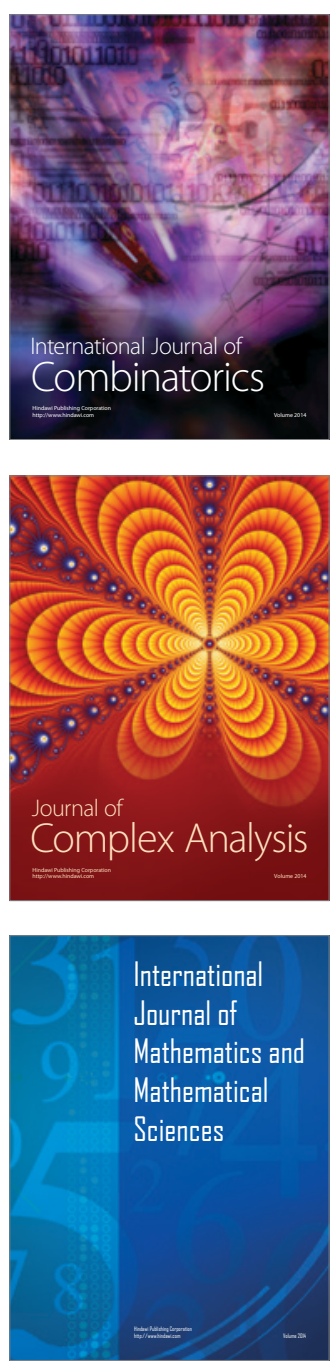
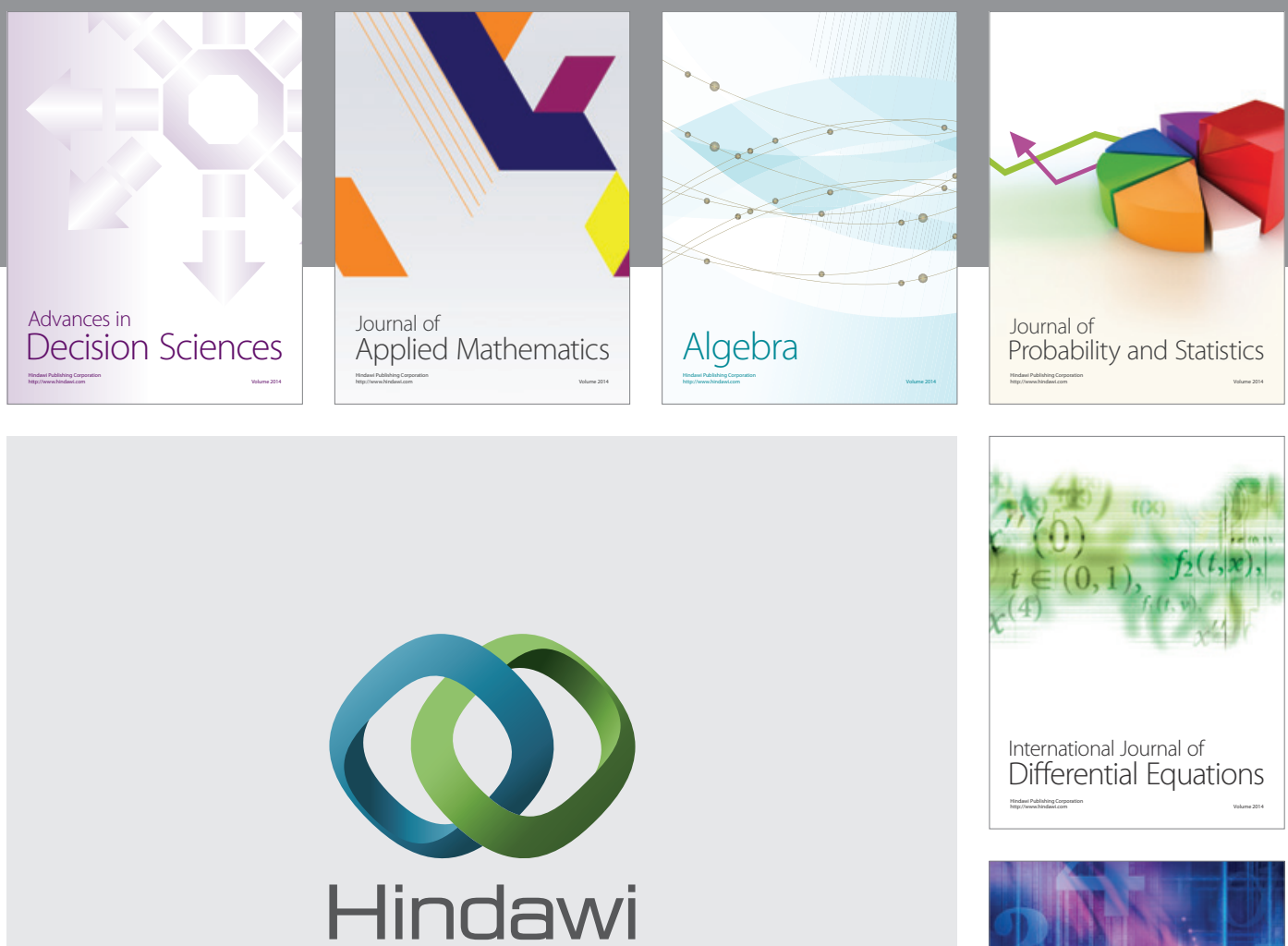

Submit your manuscripts at http://www.hindawi.com
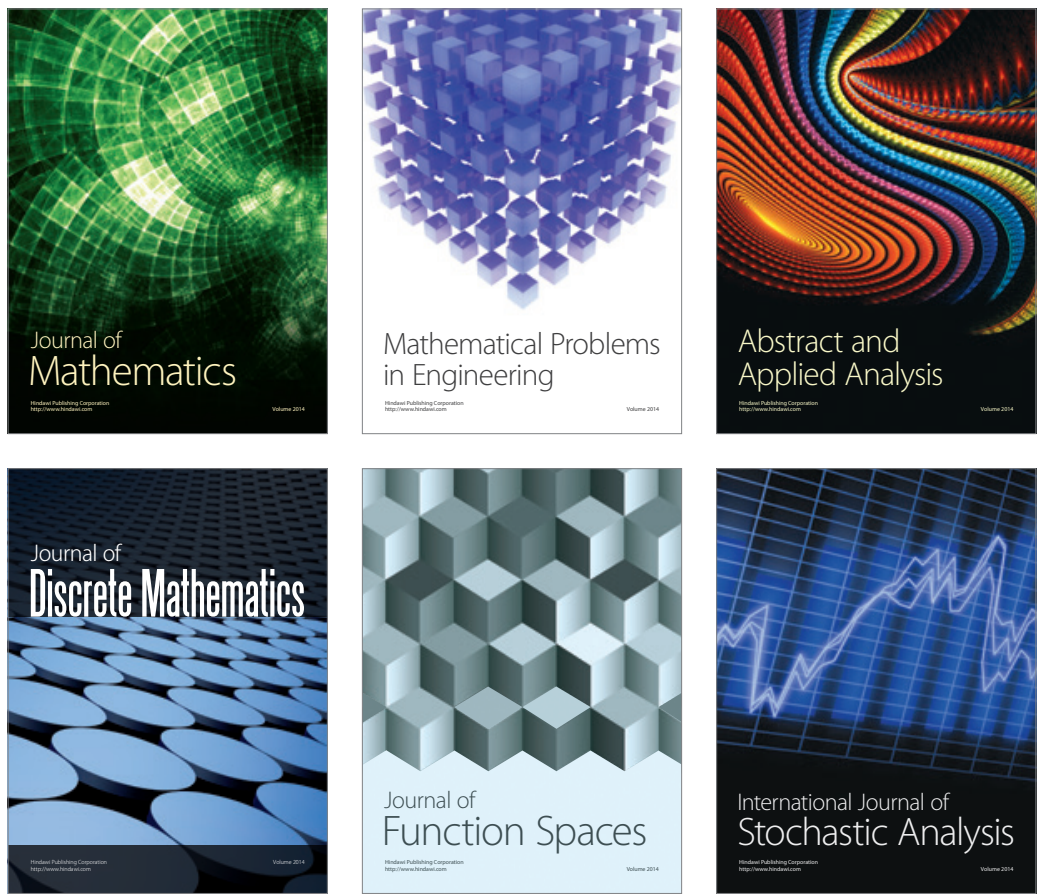

Journal of

Function Spaces

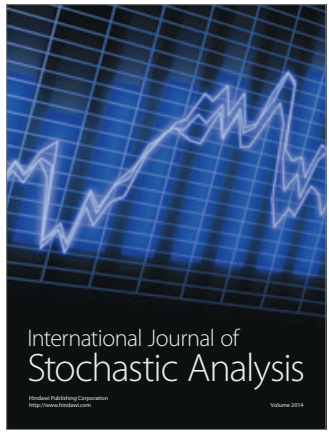

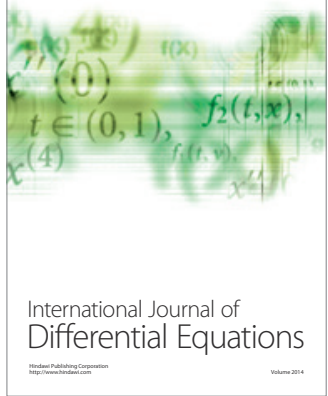
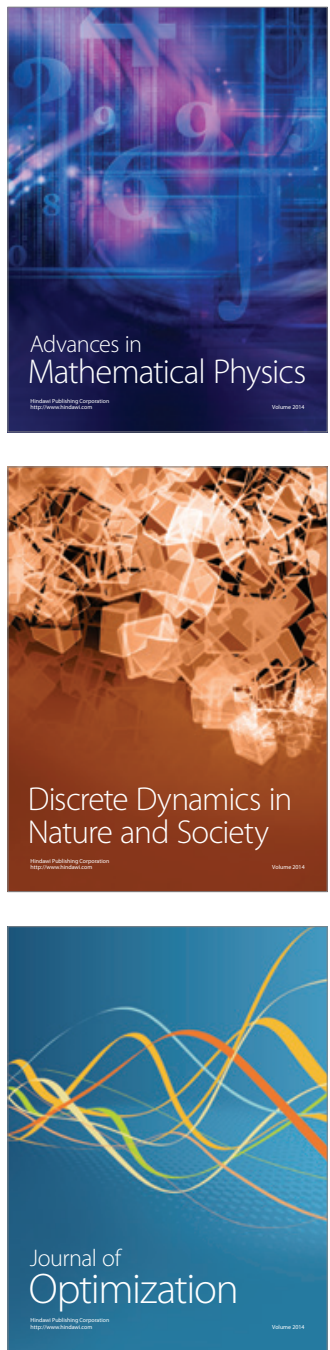